\title{
Almost-periodic solution of linearized Hasegawa-Wakatani equations with vanishing resistivity
}

\author{
SHintaro Kondo(*)
}

ABstract - In this paper we consider the zero-resistivity limit for linearized Hasegawa-Wakatani equations in a cylindrical domain when the initial data are Stepanov-almost-periodic to the axial direction. We prove two results: one is the existence and uniqueness of a strong Stepanov-almost-periodic solution to the initial boundary value problem for linearized Hasegawa-Wakatani equations with zero resistivity; another is the convergence of the solution of linearized Hasegawa-Wakatani equations established in [24] to the solution of the problem studied at the first stage as the resistivity tends to zero. In the proof we obtain two useful lemmas for Stepanov-almost-periodic functions.

Mathematics Subject Classification (2010). 35Q60; 35K45, 42A75, 42B05, $82 \mathrm{D} 10$.

KeYwords. Hasegawa-Wakatani equations, Hasegawa-Mima equation, drift wave turbulence, Sobolev spaces, Stepanov-almost-periodic function.

\section{Introduction}

There are many kinds of instabilities in plasma phenomena, and drift wave instability is one of those. Drift wave instability is classed as microinstabilities (which is a technical term of plasma physics, [36]). It has been well known that the spatial gradients in plasma lead to the drift waves and the drift wave turbulence is a natural cause of anomalous transport from which the dramatic reduction in confinement results in tokamak. Here tokamak is the most advanced magnetic confinement device, in which an

(*) Indirizzo dell'A.: Meiji Institute for Advanced Study of Mathematical Sciences, Meiji University, 4-21-1 Nakano Nakano-ku, Tokyo 164-8525, Japan.

E-mail: kondo_s@2005.jukuin.keio.ac.jp 
axisymmetric plasma is confined by a strong magnetic field. Thereby the analysis of such drift wave turbulences is important.

In order to describe the resistive drift wave turbulence in tokamak, Hasegawa and Wakatani ([19]) proposed in 1983 the following equations for the perturbations of plasma density $n$ and the electrostatic potential $\phi$ :

$$
\left\{\begin{array}{l}
\left(\frac{\partial}{\partial t}-(\nabla \phi \times \vec{e}) \cdot \nabla\right) \Delta \phi=-\frac{c_{1}}{n^{*}} \frac{\partial^{2}}{\partial x_{3}^{2}}(\phi-n)+c_{2} \Delta^{2} \phi \\
\left(\frac{\partial}{\partial t}-(\nabla \phi \times \vec{e}) \cdot \nabla\right)\left(n+\log n^{*}\right)=-\frac{c_{1}}{n^{*}} \frac{\partial^{2}}{\partial x_{3}^{2}}(\phi-n)
\end{array}\right.
$$

(Hasegawa-Wakatani equations) from the two fluids model in a homogeneous strong magnetic field $\mathbf{B}=B_{0} \vec{e}$ and an inhomogeneous plasma equilibrium density $n^{*}=n^{*}\left(\left|x^{\prime}\right|\right)\left(x=\left(x_{1}, x_{2}, x_{3}\right)=\left(x^{\prime}, x_{3}\right)\right)$ (see, [16], [20], [29]). Here the total density $N$ is divided into equilibrium and fluctuating parts, $N=n^{*}+n^{1}$, and the normalization $e \phi / T_{e} \equiv \phi, n^{1} / n^{*} \equiv n, \omega_{c i} t \equiv t$ and $x / \rho_{s} \equiv x$ are used. Here $B_{0}$ is the strength of a magnetic field assumed to be a constant, $\vec{e}=(0,0,1), c_{1}=T_{e} /\left(e^{2} \eta \omega_{c i}\right), c_{2}=\mu /\left(\rho_{s}^{2} \omega_{c i}\right), T_{e}$ is the electron temperature, $e$ is the elementary charge, $\mu$ is the kinematic ionviscosity coefficient, $\eta$ is the resistivity, $m_{i}$ is the ion mass, $\omega_{c i}=e B_{0} / m_{i}$ is the cyclotoron frequency and $\rho_{s}=\sqrt{T_{e}} /\left(\omega_{c i} \sqrt{m_{i}}\right)$ is the ion Larmor radius. For simplicity we assume that $c_{1}$ and $c_{2}$ are positive constants.

Concerning the mathematical issue for (1.1) we have a few results. In [21] we established the existence and uniqueness of a strong solution on some time interval to the initial boundary value problems for (1.1) in a cylindrical domain when the initial data are periodic to the axial direction. In [22], [23] we proved that the solution of Hasegawa-Wakatani equations established in [21] converges strongly to that of the model equations of drift wave turbulence with zero resistivity as the resistivity tends to zero. In [24] we established the existence and uniqueness of a strong Stepanovalmost-periodic solution on some time interval to the initial boundary value problems for (1.1) in a cylindrical domain when the initial data are Stepanov-almost-periodic to the axial direction.

In advance of Hasegawa-Wakatani equations Hasegawa and Mima in 1977 ([17], [18]) proposed the equation

$$
\left(\frac{\partial}{\partial t}-(\nabla \phi \times \vec{e}) \cdot \nabla\right)\left(\Delta \phi-\phi-\log n^{*}\right)=0
$$

(Hasegawa-Mima equation) from the one fluid model under the same magnetic field and plasma equilibrium state as Hasegawa-Wakatani equa- 
tions. Concerning the mathematical results for (1.2) we refer to [21], [22] and references therein.

By differencing the first and the second equations of (1.1) and linearizing it around $(\phi, n)=(0,0)$ and by denoting $\varepsilon=1 / c_{1}$, we have

$$
\left\{\begin{array}{l}
\frac{\partial}{\partial t}(\Delta \phi-n)+(\nabla \phi \times \vec{e}) \cdot \nabla \log n^{*}=c_{2} \Delta^{2} \phi \\
\varepsilon\left(\frac{\partial n}{\partial t}-(\nabla \phi \times \vec{e}) \cdot \nabla \log n^{*}\right)=-\frac{1}{n^{*}} \frac{\partial^{2}}{\partial x_{3}^{2}}(\phi-n) .
\end{array}\right.
$$

Notice that $\partial^{2} n / \partial x_{1}^{2}$ and $\partial^{2} n / \partial x_{2}^{2}$ don't appear in the right side of $(1.3)_{2}$. In this paper it will be found that when studying the zero-resistivity limit for (1.3) with almost-periodic initial data, this anisotropy causes the unexpected difficulties. Generally when looking for almost-periodic solutions, one looks for almost-periodicity in the time variable. However in this paper we consider another problem as follows:

For given an initial electrostatic potential $\phi_{0}^{\varepsilon}$, an initial plasma density $n_{0}^{\varepsilon}$ and the background density $n^{*}=n^{*}\left(\left|x^{\prime}\right|\right)$, let $\left(\phi^{\varepsilon}, n^{\varepsilon}\right)=\left(\phi^{\varepsilon}, n^{\varepsilon}\right)(x, t)$ be a solution of the initial boundary value problem for (1.3) with $\varepsilon>0$ in $\omega \times \mathbf{R} \times(0, \infty) \equiv \Omega \times(0, \infty)$ under the initial and the boundary conditions

$$
\left\{\begin{array}{l}
\phi^{\varepsilon}(x, 0)=\phi_{0}^{\varepsilon}(x), \quad n^{\varepsilon}(x, 0)=n_{0}^{\varepsilon}(x) \text { for } x \in \Omega, \\
\phi^{\varepsilon}(x, t)=\Delta \phi^{\varepsilon}(x, t)=n^{\varepsilon}(x, t)=0 \text { for } x \in \Gamma, t>0,
\end{array}\right.
$$

when the initial data are Stepanov-almost-periodic in the direction $\vec{e}$. Here $\omega=\left\{x^{\prime}=\left(x_{1}, x_{2}\right) \in \mathbf{R}^{2}|| x^{\prime} \mid<R\right\}, \partial \omega=\left\{x^{\prime}=\left(x_{1}, x_{2}\right) \in \mathbf{R}^{2}|| x^{\prime} \mid=R\right\}$, $\Gamma=\left\{x \in \mathbf{R}^{3} \mid x^{\prime} \in \partial \omega\right\}$, and $R$ is a positive real number.

For convenience, we introduce

$$
\begin{aligned}
& \bar{f}\left(x^{\prime}\right)=\mathcal{M}\{f(x)\} \equiv \lim _{A \rightarrow \infty} \frac{1}{2 A} \int_{-A}^{A} f(x) \mathrm{d} x_{3}, \\
& \widetilde{f}(x)=f(x)-\mathcal{M} f(x) \equiv(\mathcal{I}-\mathcal{M}) f(x) .
\end{aligned}
$$

Then it is easily seen that problem (1.3), (1.4) is equivalent to the problem

$$
\left\{\begin{array}{l}
\frac{\partial}{\partial t}\left(\Delta \phi^{\varepsilon}-n^{\varepsilon}\right)+\left(\nabla \phi^{\varepsilon} \times \vec{e}\right) \cdot \nabla \log n^{*}=c_{2} \Delta^{2} \phi^{\varepsilon}, \\
\varepsilon\left(\frac{\partial \widetilde{n^{\varepsilon}}}{\partial t}-\left(\nabla \widetilde{\phi^{\varepsilon}} \times \vec{e}\right) \cdot \nabla \log n^{*}\right)=-\frac{1}{n^{*}} \frac{\partial^{2}}{\partial x_{3}^{2}}\left(\widetilde{\phi^{\varepsilon}}-\widetilde{n^{\varepsilon}}\right), \\
\frac{\partial \overline{n^{\varepsilon}}}{\partial t}-\left(\nabla \overline{\phi^{\varepsilon}} \times \vec{e}\right) \cdot \nabla \log n^{*}=0 \quad \text { for } x \in \Omega, t>0,
\end{array}\right.
$$

and (1.4). 
Putting $\varepsilon=0$ in this problem, we have

$$
\left\{\begin{array}{l}
\frac{\partial}{\partial t}\left(\Delta \phi^{0}-n^{0}\right)+\left(\nabla \phi^{0} \times \vec{e}\right) \cdot \nabla \log n^{*}=c_{2} \Delta^{2} \phi^{0}, \\
\frac{1}{n^{*}} \frac{\partial^{2}}{\partial x_{3}^{2}}\left(\widetilde{\phi^{0}}-\widetilde{n^{0}}\right)=0, \\
\frac{\partial \overline{n^{0}}}{\partial t}-\left(\nabla \overline{\phi^{0}} \times \vec{e}\right) \cdot \nabla \log n^{*}=0 \quad \text { for } x \in \Omega, t>0,
\end{array}\right.
$$

and (1.4) with $\varepsilon=0$.

The aim of this paper is to establish the unique existence of a strong Stepanov-almost-periodic solution to the problem (1.5), (1.4) with $\varepsilon=0$ when the initial data are Stepanov-almost-periodic to the magnetic field direction in the same way as in [24], and the convergence of $\left(\phi^{\varepsilon}, n^{\varepsilon}\right)$ to $\left(\phi^{0}, n^{0}\right)$ as $\varepsilon$ tends to zero, which corresponds to the vanishing resistivity of linearized Hasegawa-Wakatani equations. The basic scheme of the proof in [24] essentially consists of the following steps: i) getting the approximate solutions in the form of the Bochner-Fejér sum; ii) proving that the Bochner-Fejér sum forms a sequence bounded and equi-almost-periodic; iii) overcoming the difficulty caused by the inapplicability of the RieszFischer theorem with the help of [11], [14].

Concerning Stepanov-almost-periodic solutions of Navier-Stokes equations, we have had some results. When the external force fields are sufficiently small and Stepanov-almost-periodic in time variable, the existence and uniqueness of such solutions of the initial boundary value problem for incompressible Navier-Stokes equations were proved by Foias ([15]) in 1962 in three-dimensional case and by Prouse ([31]) in 1963 in two-dimensional case. For compressible Navier-Stokes equations, similar result was obtained by Marcati and Valli ([28]) in 1985 in three-dimensional case. The basic scheme of the proof essentially consists of the following steps ([32]): i) global existence on $[0,+\infty)$ with zero initial data; ii) global existence on $(-\infty,+\infty)$; iii) Stepanov-almost-periodicity by contradiction.

In this paper, we consider the following problems:

- Let $f, g$ be almost periodic functions, and $s, \delta \in \mathbf{R}, \delta>1$ and $\eta_{s}(x) \in$ $C^{1}(\mathbf{R})$ be a cut-off function such that $\eta_{s} \equiv 1$ on $[s, s+\delta], \eta_{s} \equiv 0$ on $(-\infty, s-\delta] \cup[s+2 \delta,+\infty), \quad 0 \leq \eta_{s}(x) \leq 1$ and $\eta_{s}^{\prime}(x+2 \delta)=-\eta_{s}^{\prime}(x)$ for $x \in[s-\delta, s]$. Then we have

$$
\int_{s-\delta}^{s+2 \delta} f^{\prime}(x) g(x) \eta_{s}(x) \mathrm{d} x=-\int_{s-\delta}^{s+2 \delta}\left\{f(x) g^{\prime}(x) \eta_{s}(x)+f(x) g(x) \eta_{s}^{\prime}(x)\right\} \mathrm{d} x .
$$


When $f, g$ are periodic functions with period $L$, the last term of above equation is zero for $2 \delta=\operatorname{Ln}(n \in \mathbf{N})$, since

$$
\int_{s-\delta}^{s+2 \delta} f(x) g(x) \eta_{s}^{\prime}(x) \mathrm{d} x=\int_{s-\delta}^{s}\{f(x) g(x)-f(x+2 \delta) g(x+2 \delta)\} \eta_{s}^{\prime}(x) \mathrm{d} x .
$$

In other cases it is not zero for any $\delta>0$. In Lemma 2.1 we obtain some estimates for it.

- It is well known that if $f$ is a periodic function with period $L$, and $\int_{0}^{L} f(x) \mathrm{d} x=0$, then the Poincaré estimate holds: $\|f\| \leq c\left\|\partial_{x} f\right\|$. In Lemma 2.2 we obtain the Poincaré estimate when $f$ is an almostperiodic function and $\bar{f}=0$.

Before describing the main theorem we introduce the function spaces and the almost periodic functions that we use in the sequel ([1], [2], [5], [7], [8], [9], [13]).

Let $\Omega$ be a domain in $\mathbf{R}^{m}(m=1,2,3, \ldots)$. By $W_{2}^{l}(\Omega)(l \in \mathbf{R}, l \geq 0)$ we denote the space of functions $u(x), x \in \Omega$, equipped with the norm

$$
\|u\|_{W_{2}^{l}(\Omega)}^{2}=\sum_{|\alpha|<l}\left\|\mathrm{D}_{x}^{\alpha} u\right\|_{L^{2}(\Omega)}^{2}+\|u\|_{\dot{W}_{2}^{l}(\Omega)}^{2},
$$

where

$$
\|u\|_{\dot{W}_{2}^{l}(\Omega)}^{2}=\left\{\begin{array}{l}
\sum_{|\alpha|=l}\left\|\mathrm{D}_{x}^{\alpha} u\right\|_{L^{2}(\Omega)}^{2} \quad \text { for } l \in \mathbf{Z}, \\
\sum_{|\alpha|=[l]} \int_{\Omega} \int_{\Omega} \frac{\left|\mathrm{D}_{x}^{\alpha} u(x)-\mathrm{D}_{y}^{\alpha} u(y)\right|^{2}}{|x-y|^{m+2(l-[l])}} \mathrm{d} x \mathrm{~d} y \quad \text { for } l \notin \mathbf{Z}
\end{array} .\right.
$$

Here $[l]$ is the integral part of $l, \alpha=\left(\alpha_{1}, \alpha_{2}, \ldots, \alpha_{m}\right)$ is a multi-index, and $\mathrm{D}_{x}^{\alpha} u=\partial^{|\alpha|} u / \partial x_{1}^{\alpha_{1}} \partial x_{2}^{\alpha_{2}} \ldots \partial x_{m}^{\alpha_{m}}$ is the generalized derivative of order $|\alpha|=\alpha_{1}+\alpha_{2}+\ldots+\alpha_{m}$. For $1 \leq p \leq \infty$, we denote by $\|\cdot\|_{L^{p}(\Omega)}$ the norm of the Lebesgue space $L^{p}(\Omega)$.

The anisotropic Sobolev-Slobodetskiǔ space $W_{2}^{l, l / 2}\left(Q_{T}\right)\left(Q_{T} \equiv \Omega \times(0, T)\right)$ is defined as $L^{2}\left(0, T ; W_{2}^{l}(\Omega)\right) \cap L^{2}\left(\Omega ; W_{2}^{l / 2}(0, T)\right)$, equipped with the norm

$$
\begin{aligned}
\|u\|_{W_{2}^{l, l / 2}\left(Q_{T}\right)}^{2} & =\|u\|_{W_{2}^{l, 0}\left(Q_{T}\right)}^{2}+\|u\|_{W_{2}^{0, l / 2}\left(Q_{T}\right)}^{2} \\
& \equiv \int_{0}^{T}\|u(t)\|_{W_{2}^{l}(\Omega)}^{2} \mathrm{~d} t+\int_{\Omega}\|u(x)\|_{W_{2}^{l / 2}(0, T)}^{2} \mathrm{~d} x .
\end{aligned}
$$


Let $X$ be a Banach space with the norm $\|\cdot\|_{X}$. By $C(X)$, we denote the space of all continuous functions on $\mathbf{R}$ with values in $X$. The function $f(x) \in C(X)$ is called almost-periodic (a.p.) if for any $\varepsilon>0$ the set

$$
E_{\varepsilon}(f) \equiv\left\{\sigma \in \mathbf{R} \mid \sup _{s \in \mathbf{R}}\|f(s+\sigma)-f(s)\|_{X} \leq \varepsilon\right\}
$$

is relatively dense in $\mathbf{R}$, that is, there exists $L=L(\varepsilon)>0$ (inclusion length) such that $E_{\varepsilon}(f) \cap(a, a+L) \neq \emptyset$ for any $a \in \mathbf{R}$. By $A P(X)$, we denote the space of all a.p. functions from $\mathbf{R}$ to $X$.

By $S^{p}(X)(1 \leq p<\infty)$, we denote the subspace of $L_{l o c}^{p}(\mathbf{R} ; X)$ equipped with the finite norm

$$
\|u\|_{S^{p}(X)}^{p} \equiv \sup _{s \in \mathbf{R}} \int_{s}^{s+1}\|u(x)\|_{X}^{p} \mathrm{~d} x .
$$

The function $f(x) \in L_{l o c}^{p}(\mathbf{R} ; X)$ is called Stepanov-almost-periodic ( $S^{p}$-a.p.) ([34], [35], [37]) if for any $\varepsilon>0$ the set

$$
E_{\varepsilon}(f) \equiv\left\{\sigma \in \mathbf{R} \mid \sup _{s \in \mathbf{R}}\left(\int_{s}^{s+1}\|f(x+\sigma)-f(x)\|_{X}^{p} \mathrm{~d} x\right)^{1 / p} \leq \varepsilon\right\}
$$

is relatively dense in $\mathbf{R}$, that is, there exists $L=L(\varepsilon)>0$ (inclusion length) such that $E_{\varepsilon}(f) \cap(a, a+L) \neq \emptyset$ for any $a \in \mathbf{R}$. By $S_{a p}^{p}(X)$, we denote the space of all $S^{p}$-a.p. functions from $\mathbf{R}$ to $X$.

Let $\omega_{T} \equiv \omega \times(0, T)$ and $l \in \mathbf{Z}, l \geq 0$. We introduce the following spaces:

$$
\begin{aligned}
\widetilde{S}^{l}(X) & =\left\{u \in S^{2}(X) \mid\|u\|_{\tilde{S}^{l}(X)}^{2} \equiv \sum_{|\alpha|=0}^{l}\left\|\mathrm{D}_{x}^{\alpha} u\right\|_{S^{2}(X)}^{2}<\infty\right\}, \\
\widetilde{S}_{a p}^{l}(X) & =\left\{u \in \widetilde{S}^{l}(X)\left|\mathrm{D}_{x}^{\alpha} u \in S_{a p}^{2}(X),\right| \alpha \mid=0,1, \ldots, l\right\} \\
\widetilde{S}^{l, l / 2}\left(\omega_{T}\right) & =\widetilde{S}^{l}\left(L^{2}\left(\omega_{T}\right)\right) \cap \widetilde{S}^{0}\left(L^{2}\left(\omega ; W_{2}^{l / 2}(0, T)\right)\right), \\
\widetilde{S}_{a p}^{l, l / 2}\left(\omega_{T}\right) & =\widetilde{S}_{a p}^{l}\left(L^{2}\left(\omega_{T}\right)\right) \cap \widetilde{S}_{a p}^{0}\left(L^{2}\left(\omega ; W_{2}^{l / 2}(0, T)\right)\right) .
\end{aligned}
$$

Moreover we define the norm 
Almost-periodic solution of linearized Hasegawa-Wakatani etc.

$$
\begin{aligned}
\|u\|_{\delta}^{2} & \equiv \sup _{s \in \mathbf{R}} \frac{1}{\delta} \int_{s}^{s+\delta}\|u(x)\|_{L^{2}(\omega)}^{2} \mathrm{~d} x, \\
\|\| u \|\left.\right|_{t, \delta} ^{2} & \equiv \sup _{s \in \mathbf{R}} \frac{1}{\delta} \int_{s}^{s+\delta}\|u(x)\|_{L^{2}(\omega \times(0, t))}^{2} \mathrm{~d} x \\
\|u\|_{\tilde{S}_{\delta}^{l}}^{2} & \equiv \sum_{|\alpha|=0}^{l}\left\|\mathrm{D}_{x}^{\alpha} u\right\|_{\delta}^{2} .
\end{aligned}
$$

For simplicity, $\nabla^{\prime} \equiv\left(\partial_{1}, \partial_{2}\right), \Delta^{\prime} \equiv \partial_{1}^{2}+\partial_{2}^{2}, \partial_{k}=\partial / \partial x_{k}$.

First we prove the following theorem on the global in time existence for problem (1.3), (1.4).

THEOREM 1.1. Let $\varepsilon$ and $T$ be any positive numbers, $n^{*}\left(\left|x^{\prime}\right|\right) \in W_{2}^{2}(\omega)$ and $n^{*}\left(\left|x^{\prime}\right|\right) \geq n_{*}$ with $n_{*}$ being a positive constant. Assume that $\left(\phi_{0}^{\varepsilon}, n_{0}^{\varepsilon}\right) \in \widetilde{S}_{a p}^{4}(\omega) \times \widetilde{S}_{a p}^{2}(\omega)$ satisfies the compatibility conditions

$$
\phi_{0}^{\varepsilon}(x)=\Delta \phi_{0}^{\varepsilon}(x)=n_{0}^{\varepsilon}(x)=0 \quad \text { for } x \in \Gamma .
$$

Then there exists a unique solution $\left(\phi^{\varepsilon}, n^{\varepsilon}\right)$ to problem (1.3), (1.4) on $[0, T]$ such that $\left(\phi^{\varepsilon}, n^{\varepsilon}\right) \in L^{2}\left(0, T ; \widetilde{S}_{a p}^{4}(\omega)\right) \times \widetilde{S}_{a p}^{2,1}\left(\omega_{T}\right), \partial \phi^{\varepsilon} / \partial t \in L^{2}\left(0, T ; \widetilde{S}_{a p}^{2}(\omega)\right)$. Here $T$ is a constant independent of $\varepsilon$.

Next, it is clear that the second equation of (1.5) implies $\widetilde{\phi^{0}}-\widetilde{n^{0}}=0$ by virtue of the almost-periodicity condition in $x_{3}$ and $\mathcal{M} \tilde{\phi}^{0}=\mathcal{M} \widetilde{n^{0}}=0$. Inserting this into (1.5), (1.4) with $\varepsilon=0$, we have

$$
\left\{\begin{array}{l}
\frac{\partial}{\partial t}\left(\Delta \phi^{0}-\phi^{0}+\overline{\phi^{0}}\right)+\left(\nabla \widetilde{\phi^{0}} \times \vec{e}\right) \cdot \nabla \log n^{*}=c_{2} \Delta^{2} \phi^{0}, \\
\frac{\partial \overline{n^{0}}}{\partial t}-\left(\nabla \overline{\phi^{0}} \times \vec{e}\right) \cdot \nabla \log n^{*}=0 \text { for } x \in \Omega, t>0, \\
\phi^{0}(x, 0)=\phi_{0}^{0}(x) \text { for } x \in \Omega, \\
\overline{n^{0}}\left(x^{\prime}, 0\right)=\overline{n_{0}^{0}}\left(x^{\prime}\right) \text { for } x^{\prime} \in \omega, \\
\phi^{0}(x, t)=\Delta \phi^{0}(x, t)=0 \text { for } x \in \Gamma, t>0, \\
\overline{n^{0}}\left(x^{\prime}, t\right)=0 \text { for } x^{\prime} \in \partial \omega, t>0 .
\end{array}\right.
$$

It is to be noted that under an additional conditions $\overline{n_{0}^{0}}\left(x^{\prime}\right)=0$ and $\log n^{*}\left(x^{\prime}\right)=$ const., the equations of (1.7) is similar to the linearized 
equations of the Hasegawa-Mima equation (1.2) with an higher order correction term.

Second we prove the following theorem on the global in time existence for problem (1.7).

THeorem 1.2. Let $T$ be any positive number. Assume that $\left(\phi_{0}^{0}, \overline{n_{0}^{0}}\right) \in$ $\widetilde{S}_{a p}^{4}(\omega) \times W_{2}^{3}(\omega)$ satisfies the compatibility conditions $(1.6)$ with $\varepsilon=0$. Then there exists a unique solution $\left(\phi^{0}, \overline{n^{0}}\right)$ to the problem $(1.7)$ on $[0, T]$ such that $\left(\phi^{0}, \overline{n^{0}}\right) \in L^{2}\left(0, T ; \widetilde{S}_{a p}^{4}(\omega)\right) \times L^{\infty}\left(0, T ; W_{2}^{3}(\omega)\right), \partial \phi^{0} / \partial t \in L^{2}\left(0, T ; \widetilde{S}_{a p}^{2}(\omega)\right)$, $\partial \overline{n^{0}} / \partial t \in L^{2}\left(0, T ; W_{2}^{2}(\omega)\right)$.

For this solution $\phi^{0}$ let $n^{0}(x, t)=\phi^{0}(x, t)-\overline{\phi^{0}}\left(x^{\prime}, t\right)$ and $n_{0}^{0}(x)=\phi_{0}^{0}(x)-$ $\overline{\phi^{0}}\left(x^{\prime}, 0\right)$. Then it is easily seen that $\left(\phi^{0}, n^{0}\right)$ satisfy (1.5) and (1.4) with $\varepsilon=0$.

Finally we prove the following main result.

THeOREM 1.3. Let T be any positive number, $\left(\phi^{\varepsilon}, n^{\varepsilon}\right)$ and $\left(\phi^{0}, n^{0}\right)$ be the solutions established in Theorems 1.1 and 1.2, respectively. If the initial data $\left(\phi_{0}^{\varepsilon}, n_{0}^{\varepsilon}\right) \rightarrow\left(\phi_{0}^{0}, n_{0}^{0}\right)$ as $\varepsilon \rightarrow 0$ in $\widetilde{S}^{3}(\omega) \times \widetilde{S}^{2}(\omega)$, then as $\varepsilon \rightarrow 0,\left(\phi^{\varepsilon}, n^{\varepsilon}\right) \rightarrow$ $\left(\phi^{0}, n^{0}\right)$ in $L^{2}\left(0, T ; \widetilde{S}^{4}(\omega)\right) \times \widetilde{S}^{2,0}\left(\omega_{T}\right), \Delta \phi^{\varepsilon}-n^{\varepsilon} \rightarrow \Delta \phi^{0}-n^{0}$ in $\widetilde{S}^{0,1}\left(\omega_{T}\right)$ and $\overline{n^{\varepsilon}} \rightarrow \overline{n^{0}}$ in $\widetilde{S}^{0,1}\left(\omega_{T}\right)\left(\omega_{T} \equiv \omega \times(0, T)\right)$ on $[0, T]$.

This paper is organized as follows. In $\S 2$ we prove Theorem 1.1 from our result in [24] and the a priori estimates for problem (1.3), (1.4). In $\S 3$ Theorem 1.2 is proved through the local in time existence and a priori estimates in the same way as in [24]. In $\S 4$ we give a proof of Theorem 1.3 by virtue of a priori estimates, Theorems 1.1 and 1.2.

\section{Proof of Theorem 1.1}

For the initial boundary value problem (1.1) for $x \in \Omega, t>0$ and (1.4), we have the following theorem on the local in time existence in [24]:

THeorem 2.1. Let $n^{*}\left(\left|x^{\prime}\right|\right) \in W_{2}^{2}(\omega)$ satisfy $n^{*}\left(\left|x^{\prime}\right|\right) \geq n_{*}$ with a positive constant $n_{*}$. Assume that $\left(\phi_{0}, n_{0}\right) \in \widetilde{S}_{a p}^{4}(\omega) \times \widetilde{S}_{a p}^{2}(\omega)$ satisfies the compatibility conditions (1.6). Then there exists a unique solution $(\phi, n)$ to problem (1.1) for $x \in \Omega, t>0$ and (1.4) on some interval $\left[0, T^{*}\right]$ such that $(\phi, n) \in L^{2}\left(0, T^{*} ; \widetilde{S}_{a p}^{4}(\omega)\right) \times \widetilde{S}_{a p}^{2,1}\left(\omega_{T^{*}}\right), \partial \phi / \partial t \in L^{2}\left(0, T^{*} ; \widetilde{S}_{a p}^{2}(\omega)\right)$.

Since (1.3) are linear equations, we can easily prove the following theorem on the global in time existence: 
Proposition 2.1. Let $n^{*}\left(\left|x^{\prime}\right|\right) \in W_{2}^{2}(\omega)$ satisfy $n^{*}\left(\left|x^{\prime}\right|\right) \geq n_{*}$ with a positive constant $n_{*}$. Assume that $\left(\phi_{0}, n_{0}\right) \in \widetilde{S}_{a p}^{4}(\omega) \times \widetilde{S}_{a p}^{2}(\omega)$ satisfies the compatibility conditions (1.6). Then for any positive number $T^{* *}$ there exists a unique solution $(\phi, n)$ to problem (1.3), (1.4) on $\left[0, T^{* *}\right]$ such that $(\phi, n) \in L^{2}\left(0, T^{* *} ; \widetilde{S}_{a p}^{4}(\omega)\right) \times \widetilde{S}_{a p}^{2,1}\left(\omega_{T^{* *}}\right), \partial \phi / \partial t \in L^{2}\left(0, T^{* *} ; \widetilde{S}_{a p}^{2}(\omega)\right)$.

We denote the solution $(\phi, n)$ established in Proposition 2.1. in the case of $c_{1}=1 / \varepsilon$ by $\left(\phi^{\varepsilon}, n^{\varepsilon}\right)$. Since $T^{* *}$ in Proposition 2.1. may depend on $\varepsilon$, to complete the proof of Theorem 1.1, it is sufficient to show that $T^{* *}$ can be taken independently of $\varepsilon$.

We proceed to get a priori estimates of the solution $\left(\phi^{\varepsilon}, n^{\varepsilon}\right)$. Let it belong to $\left(L^{2}\left(0, T ; \widetilde{S}_{a p}^{4}(\omega)\right) \cap W_{2}^{1}\left(0, T ; \widetilde{S}_{a p}^{2}(\omega)\right)\right) \times \widetilde{S}_{a p}^{2,1}\left(\omega_{T}\right)$ for $T>0$. In order to get a priori estimates, in this section we use the following cut-off function $\eta_{s}$ :

- Let $s, \delta \in \mathbf{R}, \delta>1$ and $\eta_{s}\left(x_{3}\right) \in C^{1}(\mathbf{R})$ be a cut-off function such that $\eta_{s} \equiv 1$ on $[s, s+\delta], \eta_{s} \equiv 0$ on $(-\infty, s-\delta] \cup[s+2 \delta,+\infty)$, $0 \leq \eta_{s}\left(x_{3}\right) \leq 1$ and $\left|\eta_{s}^{\prime}\left(x_{3}\right)\right| \leq c / \delta,\left|\eta_{s}^{\prime \prime}\left(x_{3}\right)\right| \leq c / \delta^{2}$ with a constant $c$ independent of $\delta$ and $\eta_{s}^{\prime}\left(x_{3}+2 \delta\right)=-\eta_{s}^{\prime}\left(x_{3}\right)$ for $x_{3} \in[s-\delta, s]$.

First we prove two lemmas.

Lemma 2.1. Let $f \in S_{a p}^{1}(\omega \times(0, t)), \delta>1$. Then for any $\eta>0$ the set

$$
\left\{\delta \in \mathbf{R}|| \int_{s-\delta}^{s+2 \delta} \int_{0}^{t} \int_{\omega} f(x, \tau) \eta_{s}^{\prime}\left(x_{3}\right) \mathrm{d} x^{\prime} \mathrm{d} \tau \mathrm{d} x_{3} \mid \leq \eta\right\}
$$

is relatively dense in $\mathbf{R}$.

Proof. It is obvious from the inequality

$$
\begin{aligned}
& \int_{s-\delta}^{s+2 \delta} \int_{0}^{t} \int_{\omega} f(x, \tau) \eta_{s}^{\prime}\left(x_{3}\right) \mathrm{d} x^{\prime} \mathrm{d} \tau \mathrm{d} x_{3} \\
& =\int_{s-\delta}^{s} \int_{0}^{t} \int_{\omega}\left\{f(x, \tau)-f\left(x^{\prime}, x_{3}+2 \delta, \tau\right)\right\} \eta_{s}^{\prime}\left(x_{3}\right) \mathrm{d} x^{\prime} \mathrm{d} \tau \mathrm{d} x_{3} \\
& \quad \leq \frac{c(\delta+1)}{\delta} \sup _{s \in \mathbf{R}} \int_{s-1}^{s} \int_{0}^{t} \int_{\omega}\left|f(x, \tau)-f\left(x^{\prime}, x_{3}+2 \delta, \tau\right)\right| \mathrm{d} x^{\prime} \mathrm{d} \tau \mathrm{d} x_{3} .
\end{aligned}
$$


LEMma 2.2. Let $\psi \in S_{a p}^{2}(\omega), \partial_{3} \psi \in S_{a p}^{2}(\omega), \mathcal{M}\{\psi(x)\}=0$ in $L^{2}(\omega)$, and $\delta>1$. Then

$$
\int_{s-\delta}^{s+2 \delta}\left\|\psi\left(x_{3}\right)\right\|_{L^{2}(\omega)}^{2} \mathrm{~d} x_{3} \leq c_{\dagger}^{2} \int_{s-\delta}^{s+2 \delta}\left\|\partial_{3} \psi\left(x_{3}\right)\right\|_{L^{2}(\omega)}^{2} \mathrm{~d} x_{3} .
$$

Here $c_{\dagger}$ is a constant independent of $\delta$.

Proof. Let $X$ be a Hilbert space. It is well known that if $f \in$ $S_{a p}^{p}(X)(1 \leq p<\infty)$ and $f \in C(X)$, then $f \in A P(X)$ (see, p.76 of [1]). From this it is easy to see that $\psi \in A P\left(L^{2}(\omega)\right)$ since the following inequality holds

$$
\sup _{x_{3} \in \mathbf{R}}\left|\left\|\psi\left(x_{3}\right)\right\|_{L^{2}(\omega)}\right| \leq 2 \sup _{x_{3} \in \mathbf{R}}\left\{\int_{x_{3}}^{x_{3}+1}\left(\|\psi(s)\|_{L^{2}(\omega)}^{2}+\left\|\partial_{3} \psi(s)\right\|_{L^{2}(\omega)}^{2}\right) d s\right\}^{\frac{1}{2}}
$$

which is proved by contradiction (see, p. 89 of [3]).

Now we shall prove that the set $E^{*} \equiv\left\{x_{3} \in \mathbf{R} \mid\left\|\psi\left(x_{3}\right)\right\|_{L^{2}(\omega)}=0\right\}$ is relatively dense in $\mathbf{R}$. When $\left\|\psi\left(x_{3}\right)\right\|_{L^{2}(\omega)}=0$ for any $x_{3} \in \mathbf{R}$, it is trivial. In other cases, since $\psi \in A P\left(L^{2}(\omega)\right)$ and $\mathcal{M}\{\psi(x)\}=0$ in $L^{2}(\omega)$, we can easily prove by contradiction that there exists $y_{1} \in \mathbf{R}$ such that $\left\|\psi\left(y_{1}\right)\right\|_{L^{2}(\omega)}>0$, and there exists $y_{2} \in \mathbf{R}$ such that $\left\|\psi\left(y_{2}\right)\right\|_{L^{2}(\omega)}<0$. Hence from the intermediate value theorem, we have that there exists $y_{3} \in\left[\min \left\{y_{1}, y_{2}\right\}\right.$, $\left.\max \left\{y_{1}, y_{2}\right\}\right]$ such that $\left\|\psi\left(y_{3}\right)\right\|_{L^{2}(\omega)}=0$. From these and $\psi \in A P\left(L^{2}(\omega)\right)$, we can obtain that the set $E^{*}$ is relatively dense in $\mathbf{R}$.

Since the set $E^{*}$ is relatively dense in $\mathbf{R}$, we can introduce covering $\left\{K_{i}\right\}_{i=1}^{n}$ of $[s-\delta, s+2 \delta]$ satisfying $[s-\delta, s+2 \delta]=\cup_{i=1}^{n} K_{i}, K_{i} \cup K_{j}=\emptyset(i \neq j)$ and for any $i(i=1,2,3, \ldots, n)$ there exists $y_{i} \in K_{i}$ such that $\left\|\psi\left(y_{i}\right)\right\|_{L^{2}(\omega)}=0$. Let $c_{\dagger} \equiv \max \left|K_{i}\right|$. Then we have for $x_{3} \in K_{i}$,

$$
|\psi(x)|=\left|\int_{y_{i}}^{x_{3}} \partial_{3} \psi(x) \mathrm{d} x_{3}\right| \leq \sqrt{c_{\dagger}} \sqrt{\int_{K_{i}}\left|\partial_{3} \psi(x)\right|^{2} \mathrm{~d} x_{3} .}
$$

From this we have

$$
\begin{aligned}
& \int_{s-\delta}^{s+2 \delta}\left\|\psi\left(x_{3}\right)\right\|_{L^{2}(\omega)}^{2} \mathrm{~d} x_{3}=\sum_{i=1}^{n} \int_{K_{i}}\left\|\psi\left(x_{3}\right)\right\|_{L^{2}(\omega)}^{2} \mathrm{~d} x_{3} \\
& \leq \sum_{i=1}^{n} c_{\dagger}^{2} \int_{K_{i}}\left\|\partial_{3} \psi\left(x_{3}\right)\right\|_{L^{2}(\omega)}^{2} \mathrm{~d} x_{3}=c_{\dagger}^{2} \int_{s-\delta}^{s+2 \delta}\left\|\partial_{3} \psi\left(x_{3}\right)\right\|_{L^{2}(\omega)}^{2} \mathrm{~d} x_{3} .
\end{aligned}
$$


It is easily seen that (1.3) is equivalent to

$$
\left\{\begin{array}{l}
\frac{\partial}{\partial t}\left(\Delta \widetilde{\phi^{\varepsilon}}-\widetilde{n^{\varepsilon}}\right)+\left(\nabla \widetilde{\phi^{\varepsilon}} \times \vec{e}\right) \cdot \nabla \log n^{*}=c_{2} \Delta^{2} \widetilde{\phi^{\varepsilon}}, \\
\varepsilon\left(\frac{\partial \widetilde{n^{\varepsilon}}}{\partial t}-\left(\nabla \widetilde{\phi^{\varepsilon}} \times \vec{e}\right) \cdot \nabla \log n^{*}\right)=-\frac{1}{n^{*}} \frac{\partial^{2}}{\partial x_{3}^{2}}\left(\widetilde{\phi^{\varepsilon}}-\widetilde{n^{\varepsilon}}\right), \\
\frac{\partial \Delta \overline{\phi^{\varepsilon}}}{\partial t}=c_{2} \Delta^{2} \overline{\phi^{\varepsilon}}, \\
\frac{\partial \overline{n^{\varepsilon}}}{\partial t}-\left(\nabla \overline{\phi^{\varepsilon}} \times \vec{e}\right) \cdot \nabla \log n^{*}=0 .
\end{array}\right.
$$

Next we prove

Lemma 2.3. Let $c_{\star} \equiv \sup _{x^{\prime} \in \omega}\left|\nabla \log n^{*}\left(\left|x^{\prime}\right|\right)\right|$, and $\delta>3 c_{\dagger}^{2}\left(c_{\dagger}\right.$ is a constant of Lemma 2.2). For any $t \in[0, T], \eta>0$ the set

$$
\begin{gathered}
\left\{\delta \in \mathbf{R}\left|\varepsilon\left(\left\|\nabla \widetilde{\phi^{\varepsilon}}(t)\right\|_{\delta}^{2}+\left\|\widetilde{n^{\varepsilon}}(t)\right\|_{\delta}^{2}+\left.\left\|\Delta \widetilde{\phi^{\varepsilon}}\right\|\right|_{t, \delta} ^{2}\right)+\left\|\partial_{3}\left(\widetilde{\phi^{\varepsilon}}-\widetilde{n^{\varepsilon}}\right)\right\|\right|_{t, \delta}^{2}\right. \\
\left.\leq \varepsilon c\left(\left\|\nabla \widetilde{\phi_{0}^{\varepsilon}}\right\|_{\delta}^{2}+\left\|\widetilde{n_{0}^{\varepsilon}}\right\|_{\delta}^{2}+\frac{\eta}{\delta}\right) \mathrm{e}^{c c_{\star} t}\right\}
\end{gathered}
$$

is relatively dense in $\mathbf{R}$. Here $c$ is a constant independent of $\varepsilon$ and $\delta$, but may depends on $c_{\star}$.

Proof. Multiplying (2.1) by $\phi^{\varepsilon} \eta_{s}$ and integrating over $\Omega^{s} \equiv \omega \times$ $(s-\delta, s+2 \delta)$, we have, by virtue of the integration by parts,

$$
\begin{aligned}
\frac{1}{2} \frac{\mathrm{d}}{\mathrm{d} t} \| & \nabla \widetilde{\phi^{\varepsilon}}(t) \sqrt{\eta_{s}}\left\|_{L^{2}\left(\Omega^{s}\right)}^{2}+c_{2}\right\| \Delta \widetilde{\phi^{\varepsilon}}(t) \sqrt{\eta_{s}} \|_{L^{2}\left(\Omega^{s}\right)}^{2} \\
& +\int_{\Omega^{s}} \partial_{t} \widetilde{n^{\varepsilon}}(x, t) \widetilde{\phi^{\varepsilon}}(x, t) \eta_{s}(x) \mathrm{d} x=\int_{\Omega^{s}}\{\cdots\} \eta_{s}^{\prime}\left(x_{3}\right) \mathrm{d} x .
\end{aligned}
$$

Multiplying $(2.1)_{2}$ by $\left(\widetilde{\phi^{\varepsilon}}-\widetilde{n^{\varepsilon}}\right) \eta_{s}$ and integrating over $\Omega^{s}$, we have

$$
\begin{gathered}
\varepsilon \frac{1}{2} \frac{\mathrm{d}}{\mathrm{d} t}\left\|\widetilde{n^{\varepsilon}}(t) \sqrt{\eta_{s}}\right\|_{L^{2}\left(\Omega^{s}\right)}^{2}+\left\|\partial_{3}\left(\widetilde{\phi^{\varepsilon}}-\widetilde{n^{\varepsilon}}\right)(t) \sqrt{\frac{\eta_{s}}{n^{*}}}\right\|_{L^{2}\left(\Omega^{s}\right)}^{2} \\
-\varepsilon \int_{\Omega^{s}} \partial_{t} \widetilde{n^{\varepsilon}}(x, t) \widetilde{\phi^{\varepsilon}}(x, t) \eta_{s}\left(x_{3}\right) \mathrm{d} x
\end{gathered}
$$




$$
\begin{gathered}
=-\varepsilon \int_{\Omega^{s}}\left(\nabla \widetilde{\phi^{\varepsilon}}(x, t) \times \vec{e}\right) \cdot \nabla \log n^{*}(x)\left(\widetilde{\phi^{\varepsilon}}-\widetilde{n^{\varepsilon}}\right)(x, t) \eta_{s}\left(x_{3}\right) \mathrm{d} x \\
-\int_{\Omega^{\varepsilon}} \frac{1}{n^{*}} \partial_{3}\left(\widetilde{\phi^{\varepsilon}}-\widetilde{n^{\varepsilon}}\right)(x, t)\left(\widetilde{\phi^{\varepsilon}}-\widetilde{n^{\varepsilon}}\right)(x, t) \eta_{s}^{\prime}\left(x_{3}\right) \mathrm{d} x \\
\leq \frac{2}{3} \varepsilon c_{\star} \delta\left(\frac{1}{\varepsilon_{1}}\left\|\nabla \widetilde{\phi^{\varepsilon}}\right\|_{\delta}^{2}+\varepsilon_{1} c_{\dagger}\left\|\partial_{3}\left(\widetilde{\phi^{\varepsilon}}-\widetilde{n^{\varepsilon}}\right)(t) \sqrt{\frac{1}{n^{*}}}\right\|_{\delta}^{2}\right) \\
+3 c_{\dagger}^{2}\left\|\partial_{3}\left(\widetilde{\phi^{\varepsilon}}-\widetilde{n^{\varepsilon}}\right)(t) \sqrt{\frac{1}{n^{*}}}\right\|_{\delta}^{2} .
\end{gathered}
$$

Here we taking $\varepsilon_{1}>0$ sufficiently small. In the right most inequality, we used Lemma 2.2 and the inequality

$$
\sup _{s \in \mathbf{R}}\|f\|_{L^{2}\left(\Omega^{s}\right)}^{2} \leq 3 \delta\|f\|_{\delta}^{2} \quad \text { for } f \in S^{2}\left(L^{2}(\omega)\right) .
$$

Adding (2.4) multiplied by $1 / \delta$ and (2.3) multiplied by $\varepsilon / \delta$ and integrating this over $[0, T]$ and taking the supremum over $s \in \mathbf{R}$, we have (2.2) with the help of Gronwall's lemma, Lemma 2.1 and the inequalities $\left\|\nabla \tilde{\phi}^{\varepsilon}\right\|_{\delta} \leq c\left\|\Delta \phi^{\varepsilon}\right\|_{\delta}$ and

$$
\|f\|_{\delta}^{2} \leq \frac{1}{\delta} \sup _{s \in \mathbf{R}}\left\|f \sqrt{\eta_{s}}\right\|_{L^{2}\left(\Omega^{s}\right)}^{2} \quad \text { for } f \in S^{2}\left(L^{2}(\omega)\right) .
$$

Next we prove

LEMMA 2.4. Let $c_{\star} \equiv \sup _{x^{\prime} \in \omega}\left|\nabla \log n^{*}\left(\left|x^{\prime}\right|\right)\right|$, and $\delta>3 c_{\dagger}^{2}\left(c_{\dagger}\right.$ is a constant of Lemma 2.2). For any $t \in[0, T], \eta>0$ the set

$$
\begin{aligned}
& \left\{\delta \in \mathbf{R} \mid \varepsilon\left(\left\|\mathrm{D}_{x}^{\alpha} \Delta \widetilde{\phi^{\varepsilon}}(t)\right\|_{\delta}^{2}+\left\|\mathrm{D}_{x}^{\alpha} \nabla \widetilde{n^{\varepsilon}}(t)\right\|_{\delta}^{2}+\|\left.\left|\mathrm{D}_{x}^{\alpha} \nabla \Delta \widetilde{\phi^{\varepsilon}}\right|\right|_{t, \delta} ^{2}\right)\right. \\
& +\left.\left\||| \mathrm{D}_{x}^{\alpha} \partial_{3} \nabla\left(\widetilde{\phi^{\varepsilon}}-\widetilde{n^{\varepsilon}}\right)\right\|\right|_{t, \delta} ^{2} \leq \varepsilon c\left(\left\|\Delta \widetilde{\phi_{0}^{\varepsilon}}\right\|_{\tilde{S}_{\delta}^{|\alpha|}}^{2}+\left\|\nabla \widetilde{n_{0}^{\varepsilon}}\right\|_{\tilde{S}_{\delta}^{|\alpha|}}^{2}+\frac{\eta}{\delta}\right) \\
& \left.+\varepsilon c\left(\left\|\nabla \widetilde{\phi_{0}^{\varepsilon}}\right\|_{\delta}^{2}+\left\|\widetilde{n_{0}^{\varepsilon}}\right\|_{\delta}^{2}+\frac{\eta}{\delta}\right) \mathrm{e}^{c c_{\star} t}\right\}
\end{aligned}
$$

is relatively dense in $\mathbf{R}(|\alpha|=0,1)$. Here $\mathrm{c}$ is a constant independent of $\varepsilon$ and $\delta$, but may depends on $c_{\star}$. 
Proof. In the similar way to Lemma 2.3, multiplying $(2.1)_{1}$ and $(2.1)_{2}$ by $\Delta \widetilde{\phi^{\varepsilon}} \eta_{s}$ and $\Delta\left(\widetilde{\phi^{\varepsilon}}-\widetilde{n^{\varepsilon}}\right) \eta_{s}$, respectively, and integrating over $\Omega^{s}$, we have

$$
\begin{aligned}
\varepsilon\left(\frac{1}{2} \frac{\mathrm{d}}{\mathrm{d} t}\left(\left\|\nabla \widetilde{n^{\varepsilon}}(t) \sqrt{\eta_{s}}\right\|_{L^{2}\left(\Omega^{s}\right)}^{2}+\left\|\Delta \widetilde{\phi^{\varepsilon}}(t) \sqrt{\eta_{s}}\right\|_{L^{2}\left(\Omega^{s}\right)}^{2}\right)\right. & \left.+c_{2}\left\|\nabla \Delta \widetilde{\phi^{\varepsilon}}(t) \sqrt{\eta_{s}}\right\|_{L^{2}\left(\Omega^{s}\right)}^{2}\right)+\left\|\partial_{3} \nabla\left(\widetilde{\phi^{\varepsilon}}-\widetilde{n^{\varepsilon}}\right)(t) \sqrt{\frac{\eta_{s}}{n^{*}}}\right\|_{L^{2}\left(\Omega^{s}\right)}^{2} \\
\leq \varepsilon & \delta c_{\star} c\left\|\Delta \widetilde{\phi^{\varepsilon}}\right\|_{\delta}^{2}+C_{1}\left(\delta, c_{\star}, c_{\dagger}, \varepsilon_{1}\right)\left\|\partial_{3} \nabla\left(\widetilde{\phi^{\varepsilon}}-\widetilde{n^{\varepsilon}}\right)(t) \sqrt{\frac{1}{n^{*}}}\right\|_{\delta}^{2} \\
& +C_{2}\left(\delta, c_{\star}, c_{\dagger}, \frac{1}{\varepsilon_{1}}\right)\left\|\partial_{3}\left(\widetilde{\phi^{\varepsilon}}-\widetilde{n^{\varepsilon}}\right)(t)\right\|_{\delta}^{2}+\varepsilon \int_{\Omega^{s}}\{\cdots\} \eta_{s}^{\prime}\left(x_{3}\right) \mathrm{d} x
\end{aligned}
$$

where $\varepsilon_{1}>0, C_{1}, C_{2}$ are positive constants depending on $\delta, c_{\star}, c_{\dagger}, \varepsilon_{1}$. If we take $\delta$ sufficiently large and $\varepsilon_{1}$ sufficiently small, then $C_{1}<\delta$. In the right most inequality, we used Lemma 2.2 and (2.5) and the inequality $\left\|\nabla \widetilde{\phi^{\varepsilon}}\right\|_{\delta} \leq c\left\|\Delta \widetilde{\phi^{\varepsilon}}\right\|_{\delta}$. Integrating above inequality over [0,T] and taking the supremum over $s \in \mathbf{R}$, we have (2.7) $(|\alpha|=0)$ with the help of Lemma 2.1 and (2.2).

In the similar way to Lemma 2.3, multiply $(2.1)_{1}$ by $\Delta^{2} \widetilde{\phi}^{\varepsilon} \eta_{s}$ and integrate it over $\Omega^{s}$, operate Laplacian to $(2.1)_{2}$, multiply it by $\Delta\left(\widetilde{\phi}^{\varepsilon}-\widetilde{n}^{\varepsilon}\right) \eta_{s}$ and integrate it over $\Omega^{s}$. From these we have

$$
\begin{aligned}
\varepsilon\left(\frac{1}{2} \frac{\mathrm{d}}{\mathrm{d} t}\left(\left\|\Delta \widetilde{n^{\varepsilon}}(t) \sqrt{\eta_{s}}\right\|_{L^{2}\left(\Omega^{s}\right)}^{2}+\left\|\nabla \Delta \widetilde{\phi^{\varepsilon}}(t) \sqrt{\eta_{s}}\right\|_{L^{2}\left(\Omega^{s}\right)}^{2}\right)\right. \\
\left.\quad+c_{2}\left\|\Delta^{2} \widetilde{\phi^{\varepsilon}}(t) \sqrt{\eta_{s}}\right\|_{L^{2}\left(\Omega^{s}\right)}^{2}\right)+\left\|\partial_{3} \Delta\left(\widetilde{\phi^{\varepsilon}}-\widetilde{n^{\varepsilon}}\right)(t) \sqrt{\frac{\eta_{s}}{n^{*}}}\right\|_{L^{2}\left(\Omega^{s}\right)}^{2} \\
\leq \varepsilon C_{1}\left(\delta, c_{\star \star}, \frac{1}{\varepsilon_{1}}\right)\left(\left\|\Delta \widetilde{\phi^{\varepsilon}}\right\|_{\delta}^{2}+\left\|\nabla \Delta \widetilde{\phi^{\varepsilon}}\right\|_{\delta}^{2}\right)+\varepsilon \int_{\Omega^{s}}\{\cdots\} \eta_{s}^{\prime}\left(x_{3}\right) \mathrm{d} x \\
+C_{2}\left(\delta, c_{\star}, c_{\dagger}, \varepsilon_{1}\right)\left\|\partial_{3} \Delta\left(\widetilde{\phi^{\varepsilon}}-\widetilde{n^{\varepsilon}}\right)(t) \sqrt{\frac{1}{n^{*}}}\right\|_{\delta}^{2} \\
+C_{3}\left(\delta, c_{\star}, c_{\dagger}, \frac{1}{\varepsilon_{1}}\right)\left(\left\|\partial_{3} \nabla\left(\widetilde{\phi^{\varepsilon}}-\widetilde{n^{\varepsilon}}\right)(t)\right\|_{\delta}^{2}+\left\|\partial_{3}\left(\widetilde{\phi^{\varepsilon}}-\widetilde{n^{\varepsilon}}\right)(t)\right\|_{\delta}^{2}\right),
\end{aligned}
$$


where $\varepsilon_{1}>0, c_{\star \star} \equiv \sum_{|\alpha| \leq 2} \sup _{x^{\prime} \in \omega}\left|\mathrm{D}_{x^{\prime}}^{\alpha} \nabla \log n^{*}\left(\left|x^{\prime}\right|\right)\right|, C_{1}$ is a positive constant depending on $\delta, c_{\star \star}, \varepsilon_{1}$, and $C_{2}, C_{3}$ are positive constants depending on $\delta, c_{\star}, c_{\dagger}$, $\varepsilon_{1}$. If we take $\delta$ sufficiently large and $\varepsilon_{1}$ sufficiently small, then $C_{2}<\delta$. In the right most inequality, we used Lemma 2.2, (2.5) and the inequality $\left\|\nabla \widetilde{\phi}^{\varepsilon}\right\|_{\delta} \leq c\left\|\Delta \widetilde{\phi^{\varepsilon}}\right\|_{\delta}$. Integrating above inequality over [0,T] and taking the supremum over $s \in \mathbf{R}$, we have (2.7) $(|\alpha|=1)$ with the help of Lemma 2.1 and (2.2), (2.6), (2.7) $(|\alpha|=0)$.

The proof of the following lemma is easy, hence we omit it.

Lemma 2.5. Let $c_{\star} \equiv \sup _{x^{\prime} \in \omega}\left|\nabla \log n^{*}\left(\left|x^{\prime}\right|\right)\right|$. For any $t \in[0, T]$

$$
\begin{aligned}
& \left\|\mathrm{D}_{x^{\prime}}^{\alpha} \nabla \overline{\phi^{\varepsilon}}(t)\right\|_{L^{2}(\omega)}^{2} \leq\left\|\mathrm{D}_{x^{\prime}}^{\alpha} \nabla \overline{\phi_{0}^{\varepsilon}}\right\|_{L^{2}(\omega)}^{2} \mathrm{e}^{-c t}, \\
& \int_{0}^{t}\left\|\mathrm{D}_{x^{\prime}}^{\alpha} \Delta \overline{\phi^{\bar{\varepsilon}}}(\tau)\right\|_{L^{2}(\omega)} \mathrm{d} \tau \leq\left\|\mathrm{D}_{x^{\prime}}^{\alpha} \nabla \overline{\phi_{0}^{\varepsilon}}\right\|_{L^{2}(\omega)}^{2}, \quad|\alpha|=0,1,2, \\
& \left\|\overline{n^{\varepsilon}}(t)\right\|_{L^{2}(\omega)}^{2} \leq\left\|\overline{n_{0}^{\varepsilon}}\right\|_{L^{2}(\omega)}^{2}+c_{\star} c t\left\|\nabla \overline{\phi_{0}^{\varepsilon}}\right\|_{L^{2}(\omega)}^{2}, \\
& \left\|\mathrm{D}_{x^{\prime}}^{\alpha} \overline{n^{\varepsilon}}(t)\right\|_{L^{2}(\omega)}^{2} \leq\left\|\mathrm{D}_{x^{\prime}}^{\alpha} \overline{n_{0}^{\varepsilon}}\right\|_{L^{2}(\omega)}^{2}+c_{\star} c t\left\|\mathrm{D}_{x^{\prime}}^{\alpha} \overline{\phi_{0}^{\varepsilon}}\right\|_{L^{2}(\omega)}^{2}, \quad|\alpha|=1,2,3, \\
& \left\|\frac{\partial \mathrm{D}_{x^{\prime}}^{\alpha} \overline{n^{\varepsilon}}}{\partial t}(t)\right\|_{L^{2}(\omega)}^{2} \leq c_{\star}\left\|\mathrm{D}_{x^{\prime}}^{\alpha} \overline{\phi_{0}^{\varepsilon}}\right\|_{L^{2}(\omega)}^{2}, \quad|\alpha|=0,1,2 .
\end{aligned}
$$

Here $c$ is a constant independent of $\varepsilon$, but may depends on $c_{\star}$.

By the standard arguments based upon the a priori estimates in Lemmas 2.3-2.5 the solution can be extended up to any T. Since (1.3) are linear equations, we can easily prove Stepanov-almost-periodicity of the solution. Thus the proof of Theorem 1.1 is complete.

\section{Proof of Theorem 1.2}

The proof of Theorem 1.2 is divided into two parts. First we prove the local in time existence in the same way as in [24] in $\S 3.1$. Second we give a proof of Theorem 1.2 with the help of a priori estimates in $\S 3.2$. 


\section{1 - Local in time existence and uniqueness}

\subsection{1 - Auxiliary lemmas}

Let $X$ be a Hilbert space and $\psi \in S_{a p}^{2}(X)$. Note that for any $\xi \in \mathbf{R}$ the mean value

$$
\psi_{\xi}=\mathcal{M}\left\{\psi(x) \mathrm{e}^{-\mathrm{i} \xi x_{3}}\right\} \equiv \lim _{A \rightarrow \infty} \frac{1}{2 A} \int_{-A}^{A} \psi(x) \mathrm{e}^{-\mathrm{i} \xi x_{3}} \mathrm{~d} x_{3}
$$

exists in $X$ ([10], [38]), where $\mathrm{i}=\sqrt{-1}$.

Let $\left\{\xi_{k}\right\}_{k \in \mathbf{N}}$ be a sequence in $\mathbf{R}$ such that $\xi_{k} \neq \xi_{k^{\prime}}$ for $k \neq k^{\prime}$. For each $m \in \mathbf{N}$, it is easy to obtain

$$
\mathcal{M}\left\{\left\|\psi\left(x_{3}\right)-\sum_{k=1}^{m} \psi_{\xi_{k}} \mathrm{e}^{-\mathrm{i} \xi_{k} x_{3}}\right\|_{X}^{2}\right\}=\mathcal{M}\left\{\left\|\psi\left(x_{3}\right)\right\|_{X}^{2}\right\}-\sum_{k=1}^{m}\left\|\psi_{\xi_{k}}\right\|_{X}^{2},
$$

and hence

$$
\sum_{k=1}^{m}\left\|\psi_{\xi_{k}}\right\|_{X}^{2} \leq \mathcal{M}\left\{\left\|\psi\left(x_{3}\right)\right\|_{X}^{2}\right\}
$$

This inequality implies that for any $\varepsilon>0$ there corresponds at most a finite number of $\xi_{k}$ for which $\left\|\psi_{\xi_{k}}\right\|_{X}>\varepsilon$. From this fact it follows that every $\left\|\psi_{\xi_{k}}\right\|_{X}(\neq 0)$ belongs to one of the enumerable set of inequalities

$$
\left\|\psi_{\xi_{k}}\right\|_{X}>1, \quad \frac{1}{m} \geq\left\|\psi_{\xi_{k}}\right\|_{X}>\frac{1}{m+1} \quad(m=1,2,3, \ldots),
$$

and each of these inequalities is satisfied by at most a finite number of $\xi_{k}$. Therefore, only for at most countable $\xi \in \mathbf{R}$ the quantity $\psi_{\xi}$ is a non-zero element of $X$. We call $\sigma(\psi)=\left\{\xi \in \mathbf{R} \mid\left\|\psi_{\xi}\right\|_{X} \neq 0\right\}$ the spectrum of $\psi$, and the formal series $\sum_{\xi \in \sigma(\psi)} \psi_{\xi} \mathrm{e}^{\mathrm{i} \xi x_{3}}$ the Bohr-Fourier series of $\psi$, which is written as

$$
\psi \sim \sum_{\xi \in \sigma(\psi)} \psi_{\xi} \mathrm{e}^{\mathrm{i} \xi x_{3}}
$$

Then the following lemmas hold (see [1], [5], [12], [13]).

Lemma 3.1. If $\psi, \psi^{\prime} \in S_{\text {ap }}^{2}(X)$ have the same Bohr-Fourier series, then

$$
\left\|\psi-\psi^{\prime}\right\|_{S^{2}(X)}=0 \text {. }
$$


LEMMA 3.2. For any $\psi \in S_{a p}^{2}(X)$ Parseval's identity

$$
\mathcal{M}\left\{\left\|\psi\left(x_{3}\right)\right\|_{X}^{2}\right\}=\sum_{\xi \in \sigma(\psi)}\|\psi\|_{X}^{2}
$$

holds.

Let us consider a generalized trigonometric series

$$
\sum_{\xi \in \Lambda} a_{\xi} \mathrm{e}^{\mathrm{i} \xi x}
$$

where $\Lambda$ is a countable subset of $\mathbf{R}$ and $\left\{a_{\xi}\right\}_{\xi \in \Lambda} \subset \mathbf{C}$. Let $\left\{\gamma_{j}\right\}_{j \in \mathbf{N}}$ be a basis of $\Lambda$ ([10]). Bochner-Fejér sum $\mathcal{S}^{m}(x)$ associated with (3.1) is given by

$$
\begin{gathered}
\mathcal{S}^{m}(x)=\sum_{v_{1}=-(m !)^{2}}^{(m !)^{2}} \cdots \sum_{v_{m}=-(m !)^{2}}^{(m !)^{2}}\left(1-\frac{\left|v_{1}\right|}{(m !)^{2}}\right) \cdots\left(1-\frac{\left|v_{m}\right|}{(m !)^{2}}\right) \\
\times a_{\xi}^{*} \exp \left(\mathrm{i} \sum_{j=1}^{m} v_{j} \frac{\gamma_{j}}{m !} x\right),
\end{gathered}
$$

where for $\xi \in \Lambda$

$$
a_{\xi}^{*}= \begin{cases}a_{\xi} & \text { if } \quad \sum_{j=1}^{m} v_{j} \frac{\gamma_{j}}{m !}=\xi \\ 0 & \text { if } \quad \sum_{j=1}^{m} v_{j} \frac{\gamma_{j}}{m !} \neq \xi\end{cases}
$$

By introducing an increasing symmetric sequence $\left\{\Lambda_{m}\right\}_{m \in \mathbf{N}}$ of $\Lambda$ converging to $\Lambda$, that is, $-\Lambda_{m}=\Lambda_{m}, \Lambda_{m} \subset \Lambda_{m+1}$ and $\Lambda=\cup_{m} \Lambda_{m}, \mathcal{S}^{m}(x)$ can be written as

$$
\mathcal{S}^{m}(x)=\sum_{\xi \in \Lambda_{m}} d_{\xi}^{(m)} a_{\xi} \mathrm{e}^{\mathrm{i} \xi x}
$$

with constants $d_{\xi}^{(m)}$ satisfying $0 \leq d_{\xi}^{(m)} \leq 1$ and $\lim _{m \rightarrow \infty} d_{\xi}^{(m)}=1$. Note that $d_{\xi}^{(m)}$ depend on $\xi$ and $m$, but not on $a_{\xi}$ ([13]).

We say that $\mathcal{F} \subset S_{a p}^{p}(X)$ is $S^{p}$-equi-almost-periodic if for any $\varepsilon>0$ there exists a relatively dense subset $E_{\varepsilon}$ of $\mathbf{R}$ such that

$$
\sup _{s \in \mathbf{R}} \int_{s}^{s+1}\|f(x+\sigma)-f(x)\|_{X}^{p} \mathrm{~d} x<\varepsilon \quad \text { for } f \in \mathcal{F}, \sigma \in E_{\varepsilon} .
$$


It is well-known that Riesz-Fischer theorem does not hold for $S_{a p}^{p}(X)$ $(1 \leq p<\infty)$ ([4], [27]), while the following lemma holds true (see [11], [14]).

LEMma 3.3. A necessary and sufficient condition for a generalized trigonometric series (3.1) to be a Bohr-Fourier series of a function $f \in S_{a p}^{p}(X)(1<p<\infty)$ is that a sequence of the Bochner-Fejer sums $\left\{\mathcal{S}^{m}(x)\right\}_{m \in \mathbf{N}}$ associated with the series (3.1) is bounded in $S^{p}(X)$ and $S^{p}$ equi-almost-periodic.

\subsection{2 - Local in time existence and uniqueness}

It is easily seen that (1.7) $)_{2}$ has a unique solution $\overline{n^{0}}$ when $\overline{\phi^{0}}$ is given. Hence we consider only the problem for (1.7) $)_{1}$. The following lemmas are well-known (see, for example, [26], [30], [33]).

Lemma 3.4. Let $l \in \mathbf{R}, l \geq 0, c_{2}>0$ and $\xi \in \mathbf{R}$. Assume that $\psi_{0} \in W_{2}^{1+l}(\omega)$ satisfies the compatibility conditions up to order $\max \{[l-3 / 2], 0\}$ and $f \in W_{2}^{l, l / 2}\left(\omega_{T}\right)$ satisfies $f\left(x^{\prime}, t\right)=0$ for $x^{\prime} \in \partial \omega, t>0$. Then there exists a unique solution $\psi \in W_{2}^{2+l, 1+l / 2}\left(\omega_{T}\right)$ to problem

$$
\left\{\begin{array}{l}
\frac{\partial \psi}{\partial t}-c_{2}\left(\Delta^{\prime}-\xi^{2}\right) \psi=f \text { for } x^{\prime} \in \omega, t>0 \\
\psi\left(x^{\prime}, 0\right)=\psi_{0}\left(x^{\prime}\right) \text { for } x^{\prime} \in \omega \\
\psi\left(x^{\prime}, t\right)=0 \text { for } x^{\prime} \in \partial \omega, t>0
\end{array}\right.
$$

Moreover, this solution satisfies

$$
\|\psi\|_{W_{2}^{2+l, 1+l / 2}\left(\omega_{T}\right)} \leq c_{\xi}\left(\left\|\psi_{0}\right\|_{W_{2}^{1+l}(\omega)}+\|f\|_{W_{2}^{l, l / 2}\left(\omega_{T}\right)}\right)
$$

with $c_{\xi}$ being a positive constant depending on $\xi$.

Lemma 3.5. Let $\psi \in W_{2}^{2+l, 1+l / 2}\left(\omega_{T}\right), l \geq 0$ and $\xi \in \mathbf{R}$. Then the problem

$$
\left\{\begin{array}{l}
\left(\Delta^{\prime}-\xi^{2}\right) \phi=\psi \quad \text { for } x^{\prime} \in \omega, t>0, \\
\phi\left(x^{\prime}, t\right)=0 \text { for } x^{\prime} \in \partial \omega, t>0
\end{array}\right.
$$

has a unique solution $\phi \in L^{2}\left(0, T ; W_{2}^{4+l}(\omega)\right) \cap W_{2}^{1+l / 2}\left(0, T ; W_{2}^{2}(\omega)\right)$, which satisfies

$$
\|\phi\|_{L^{2}\left(0, T ; W_{2}^{4+l}(\omega)\right)}+\|\phi\|_{W_{2}^{1+l / 2}\left(0, T ; W_{2}^{2}(\omega)\right)} \leq c_{\xi}\|\psi\|_{W_{2}^{2+l, 1+l / 2}\left(\omega_{T}\right)}
$$

with $c_{\xi}$ being a positive constant depending on $\xi$. 
Let us fix symmetric increasing sequence $\left\{\Lambda_{m}\right\}_{m \in \mathbf{N}}$ of $\Lambda \equiv \sigma\left(\phi_{0}^{0}\right)$ converging to $\Lambda$. For $\xi \in \Lambda$ we consider problem

$$
\left\{\begin{array}{l}
\frac{\partial}{\partial t}\left(\left(\Delta^{\prime}-\xi^{2}\right) \phi_{\xi}^{0}-\phi_{\xi}^{0}+\overline{\phi_{\xi}^{0}}\right)+\left(\nabla \widetilde{\phi_{\xi}^{0}} \times \vec{e}\right) \cdot \nabla \log n^{*} \\
\quad=c_{2}\left(\Delta^{\prime}-\xi^{2}\right)^{2} \phi_{\xi}^{0} \text { for } x^{\prime} \in \omega, t>0, \\
\left.\phi_{\xi}^{0}\right|_{t=0}=\phi_{0 \xi}^{0} \text { for } x^{\prime} \in \omega, \\
\phi_{\xi}^{0}=\left(\Delta^{\prime}-\xi^{2}\right) \phi_{\xi}^{0}=0 \text { for } x^{\prime} \in \partial \omega, t>0,
\end{array}\right.
$$

where $\phi_{0 \xi}^{0}=\mathcal{M}\left\{\phi_{0}^{0} \mathrm{e}^{-\mathrm{i} \xi \mathrm{x}_{3}}\right\}$. Lemma 3.4 implies that (3.2) has a unique solution $\phi_{\xi}^{0}$. Then it is obvious that $\mathcal{S}_{\phi^{0}}^{m}=\sum_{\xi \in A_{m}} d_{\xi}^{(m)} \phi_{\xi}^{0} \mathrm{e}^{\mathrm{i} \xi x_{3}}$ is a solution of
problem

$$
\left\{\begin{array}{l}
\frac{\partial}{\partial t}\left(\Delta \mathcal{S}_{\phi^{0}}^{m}-\mathcal{S}_{\phi^{0}}^{m}+\mathcal{S}_{\phi^{0}}^{m}\right)+\left(\nabla \mathcal{S}_{\phi^{0}}^{m} \times \vec{e}\right) \cdot \nabla \log n^{*} \\
\quad=c_{2} \Delta^{2} \mathcal{S}_{\phi^{0}}^{m} \quad \text { for } x \in \Omega, t>0, \\
\left.\mathcal{S}_{\phi^{0}}^{m}\right|_{t=0}=\mathcal{S}_{\phi_{0}^{0}}^{m} \text { for } x \in \Omega, \\
\mathcal{S}_{\phi^{0}}^{m}=\Delta \mathcal{S}_{\phi^{0}}^{m}=0 \quad \text { for } x \in \Gamma, t>0
\end{array},\right.
$$

where $\mathcal{S}_{\phi_{0}^{0}}^{m}=\sum_{\xi \in \Lambda_{m}} d_{\xi}^{(m)} \phi_{0 \xi}^{0} \mathrm{e}^{\mathrm{i} \xi x_{3}}$.

The following lemma holds in the same way as in Lemmas 2.3-2.4.

LEMmA 3.6. Let $c_{\star} \equiv \sup \left|\nabla \log n^{*}\left(\left|x^{\prime}\right|\right)\right|$ and $\widetilde{\phi^{0}}=\phi^{0}-\overline{\phi^{0}}$. For any $t \in[0, T], \eta>0$ the sets

$$
\begin{gathered}
\left\{\delta \in \mathbf{R} \mid\left\|\nabla \mathcal{S}_{\phi^{0}}^{m}(t)\right\|_{\delta}^{2}+\left\|\mathcal{S}_{\widetilde{\phi}^{0}}^{m}(t)\right\|_{\delta}^{2}+\|\|_{\mathcal{S}_{\phi^{0}}^{m}}^{m}\|\|_{t, \delta}^{2}\right. \\
\left.\leq \varepsilon c\left(\left\|\nabla \mathcal{S}_{\dot{\phi}_{0}^{0}}^{m}\right\|_{\delta}^{2}+\left\|\mathcal{S}_{\widetilde{\phi}_{0}^{0}}^{m}\right\|_{\delta}^{2}+\frac{\eta}{\delta}\right) \mathrm{e}^{c c_{\star} t}\right\} \\
\left\{\delta \in \mathbf{R}\left|\left\|\mathrm{D}_{x}^{\alpha} \Delta \mathcal{S}_{\phi^{0}}^{m}(t)\right\|_{\delta}^{2}+\left\|\mathrm{D}_{x}^{\alpha} \nabla \mathcal{S}_{\widetilde{\phi}^{0}}^{m}(t)\right\|_{\delta}^{2}+\|\| \mathrm{D}_{x}^{\alpha} \nabla \Delta \mathcal{S}_{\phi^{0}}^{m}\right| \|_{t, \delta}^{2}\right. \\
\leq \varepsilon c\left(\left\|\Delta \mathcal{S}_{\phi_{0}^{0}}^{m}\right\|_{\tilde{S}_{\delta}^{|\alpha|}}^{2}+\left\|\nabla \mathcal{S}_{\widetilde{\phi}_{0}^{0}}^{m}\right\|_{\tilde{S}_{\delta}^{|\alpha|}}^{2}+\frac{\eta}{\delta}\right) \\
\left.+\varepsilon c\left(\left\|\nabla \mathcal{S}_{\phi_{0}^{0}}^{m}\right\|_{\delta}^{2}+\left\|\mathcal{S}_{\widetilde{\phi}_{0}^{0}}^{m}\right\|_{\delta}^{2}+\frac{\eta}{\delta}\right) \mathrm{e}^{c c_{\star} t}\right\}
\end{gathered}
$$


are relatively dense in $\mathbf{R}(|\alpha|=0,1)$. Here $c$ is a constant independent of $\delta$, but may depends on $c_{\star}$.

Let $\mathcal{V}_{\cdot \sigma}^{m}(x, t)=\mathcal{S}_{.}^{m}\left(x^{\prime}, x_{3}+\sigma, t\right)-\mathcal{S}_{.}^{m}\left(x^{\prime}, x_{3}, t\right)$ for any $\sigma \neq 0$. Then $\mathcal{V}_{\phi^{0} \sigma}^{m}$ satisfies

$$
\left\{\begin{array}{l}
\frac{\partial}{\partial t}\left(\Delta \mathcal{V}_{\phi^{0} \sigma}^{m}-\mathcal{V}_{\phi^{0} \sigma}^{m}+\mathcal{V}_{\phi^{0} \sigma}^{m}\right. \\
=c_{2} \Delta^{2} \mathcal{V}_{\phi^{0} \sigma}^{m} \quad \text { for } x \in \Omega, t>0, \\
\left.\mathcal{V}_{\phi^{0} \sigma}^{m}\right|_{t=0}=\mathcal{V}_{\phi_{0}^{0} \sigma}^{m} \text { for } x \in \Omega, \\
\mathcal{V}_{\phi^{0} \sigma}^{m}=\Delta \mathcal{V}_{\phi^{0} \sigma}^{m}=0 \quad \text { for } x \in \Gamma, t>0 .
\end{array}\right.
$$

The following lemma holds in the same way as in Lemma 3.6.

LEMMA 3.7. Let $c_{\star} \equiv \sup _{x^{\prime} \in \omega}\left|\nabla \log n^{*}\left(\left|x^{\prime}\right|\right)\right|$ and $\widetilde{\phi^{0}}=\phi^{0}-\overline{\phi^{0}}$. For any $t \in[0, T], \eta>0$ the sets

$$
\begin{gathered}
\left\{\delta \in \mathbf{R} \mid\left\|\nabla \mathcal{V}_{\phi^{0} \sigma}^{m}(t)\right\|_{\delta}^{2}+\left\|\mathcal{V}_{\phi_{\phi^{0} \sigma}^{m}}^{m}(t)\right\|_{\delta}^{2}+\left\|\mathcal{V}_{\phi^{0} \sigma}^{m}\right\| \|_{t, \delta}^{2}\right. \\
\left.\leq \varepsilon c\left(\left\|\nabla \mathcal{V}_{\phi_{0}^{0} \sigma}^{m}\right\|_{\delta}^{2}+\left\|\mathcal{V}_{\phi_{0}^{0} \sigma}^{m}\right\|_{\delta}^{2}+\frac{\eta}{\delta}\right) \mathrm{e}^{c c_{\star} t}\right\}, \\
\left\{\delta \in \mathbf{R} \mid\left\|\mathrm{D}_{x}^{\alpha} \Delta \mathcal{V}_{\phi^{0} \sigma}^{m}(t)\right\|_{\delta}^{2}+\left\|\mathrm{D}_{x}^{\alpha} \nabla \mathcal{V}_{\phi^{0} \sigma}^{m}(t)\right\|_{\delta}^{2}+\left\|\mathrm{D}_{x}^{\alpha} \nabla \Delta \mathcal{V}_{\phi^{0} \sigma}^{m}\right\|_{t, \delta}^{2}\right. \\
\leq \varepsilon c\left(\left\|\Delta \mathcal{V}_{\phi_{0}^{0} \sigma}^{m}\right\|_{\tilde{S}_{\delta}^{|\alpha|}}^{2}+\left\|\nabla \mathcal{V}_{\widetilde{\phi_{0}^{0} \sigma}}^{m}\right\|_{\tilde{S}_{\delta}^{|\alpha|}}^{2}+\frac{\eta}{\delta}\right) \\
\left.+\varepsilon c\left(\left\|\nabla \mathcal{V}_{\phi_{0}^{0} \sigma}^{m}\right\|_{\delta}^{2}+\left\|\mathcal{V}_{\widetilde{\phi_{0}^{0} \sigma}}^{m}\right\|_{\delta}^{2}+\frac{\eta}{\delta}\right) \mathrm{e}^{c c_{\star} t}\right\},
\end{gathered}
$$

are relatively dense in $\mathbf{R}(|\alpha|=0,1)$. Here c is a constant independent of $\delta$, but may depends on $c_{\star}$.

Now we prove that $\left\{\mathcal{S}_{\phi^{0}}^{m}\right\}_{m=1}^{\infty}$ forms a sequence bounded in $\widetilde{S}^{3,3 / 2}\left(\omega_{T}\right)$ and $\widetilde{S}^{3,3 / 2}\left(\omega_{T}\right)$-equi-almost-periodic with the help of Lemmas 3.6-3.7 and the well-known fact (see [4], [6], [11], [13])

$$
\begin{aligned}
& \left\|\mathcal{S}_{\psi}^{m}\right\|_{S^{p}(X)} \leq\|\psi\|_{S^{p}(X)}, \\
& \left\|\mathcal{S}_{\psi}^{m}-\psi\right\|_{S^{p}(X)} \rightarrow 0 \text { as } m \rightarrow \infty
\end{aligned}
$$

for any $\psi \in S_{a p}^{p}(X)$ defined on a Banach space $X(1 \leq p \leq \infty)$. 
Indeed, the boundedness of $\left\{\mathcal{S}_{\phi^{0}}^{m}\right\}_{m=1}^{\infty}$ in $\widetilde{S}^{3,3 / 2}\left(\omega_{T}\right)$ followed from

$$
\left\|\mathcal{S}_{\phi_{0}^{0}}^{m}\right\|_{\tilde{S}^{2}}^{2} \leq\left\|\phi_{0}^{0}\right\|_{\tilde{S}^{2}}^{2}
$$

directly derived from (3.4) and Lemma 3.6.

Let

$$
\Phi_{0 \sigma}^{0}(x)=\phi_{0}^{0}\left(x^{\prime}, x_{3}+\sigma\right)-\phi_{0}^{0}\left(x^{\prime}, x_{3}\right)
$$

for any $\sigma \neq 0$. It is easy to see that $\Phi_{0 \sigma \xi}^{0}=\left(\mathrm{e}^{\mathrm{i} \xi \sigma}-1\right) \phi_{0 \xi}^{0}$ and

$$
\mathcal{V}_{\phi_{0}^{0} \sigma}^{m}(x)=\mathcal{S}_{\Phi_{0 \sigma}^{0}}^{m}(x) \text {. }
$$

Then (3.4) yields

$$
\left\|\mathcal{V}_{\phi_{0}^{0} \sigma}^{m}\right\|_{\tilde{S}^{2}}^{2} \leq\left\|\Phi_{0 \sigma}^{0}\right\|_{\tilde{S}^{2}}^{2} .
$$

From this we find that $\mathcal{S}_{\phi^{0}}^{m}$ is $\widetilde{S}^{3,3 / 2}\left(\omega_{T}\right)$-equi-almost-periodic by virtue of Lemmas 3.7.

Lemmas 3.3 implies that $\phi^{0}$ belongs to $\widetilde{S}_{a p}^{3,3 / 2}\left(\omega_{T}\right)$. Moreover $\phi^{0}$ is unique in the same class according to Lemma 3.1. Therefore from (3.5), we have

$$
\mathcal{S}_{\phi^{0}}^{m} \rightarrow \phi^{0} \quad \text { in } \widetilde{S}^{3,3 / 2}\left(\omega_{T}\right) \quad \text { as } m \rightarrow \infty .
$$

Since

$$
\mathcal{S}_{\phi_{0}^{0}}^{m} \rightarrow \phi_{0}^{0} \quad \text { in } \widetilde{S}^{2}(\omega) \quad \text { as } m \rightarrow \infty
$$

follows from (3.5), we conclude from (3.3) and (3.6), that this $\phi^{0}$ is a solution of the problem for $(1.7)_{1}$. Here the strong convergence of Galerkin approximations are obtained by using property of linear equations (p. 79 of [25]). Thus the proof of local in time existence and uniqueness is complete.

\section{$3.2-A$ priori estimates}

In the similar way as in Lemmas 2.3-2.5 we can show the following a priori estimates of the solution $\left(\phi^{0}, \overline{n^{0}}\right)$ established in $\S 3.1$. Let $T$ be an arbitrary positive number and $\left(\phi^{0}, \overline{n^{0}}\right)$ be a solution of problem (1.7) belonging to $\left(L^{2}\left(0, T ; \widetilde{S}^{4}(\omega)\right) \cap W_{2}^{1}\left(0, T ; \widetilde{S}^{2}(\omega)\right)\right) \times\left(L^{\infty}\left(0, T ; W_{2}^{3}(\omega)\right) \cap W_{2}^{1}\left(0, T ; W_{2}^{2}(\omega)\right)\right)$.

LEMMA 3.8. Let $c_{\star} \equiv \sup \left|\nabla \log n^{*}\left(\left|x^{\prime}\right|\right)\right|$ and $\widetilde{\phi^{0}}=\phi^{0}-\overline{\phi^{0}}$. For any $t \in[0, T], \eta>0$ the sets 


$$
\begin{gathered}
\left\{\delta \in \mathbf{R} \mid\left\|\nabla \widetilde{\phi^{0}}(t)\right\|_{\delta}^{2}+\left\|\widetilde{\phi^{0}}(t)\right\|_{\delta}^{2}+\left\|\Delta \widetilde{\phi^{0}}\right\|_{t, \delta}^{2}\right. \\
\left.\leq \varepsilon c\left(\left\|\nabla \widetilde{\phi_{0}^{0}}\right\|_{\delta}^{2}+\left\|\widetilde{\phi_{0}^{0}}\right\|_{\delta}^{2}+\frac{\eta}{\delta}\right) \mathrm{e}^{c c_{\star} t}\right\}, \\
\left\{\delta \in \mathbf{R} \mid\left\|\mathrm{D}_{x}^{\alpha} \Delta \widetilde{\phi^{0}}(t)\right\|_{\delta}^{2}+\left\|\mathrm{D}_{x}^{\alpha} \nabla \widetilde{\phi^{0}}(t)\right\|_{\delta}^{2}+\left\|\mathrm{D}_{x}^{\alpha} \nabla \Delta \widetilde{\phi^{0}}\right\|_{t, \delta}^{2}\right. \\
\leq \varepsilon c\left(\left\|\Delta \widetilde{\phi_{0}^{0}}\right\|_{\tilde{S}_{\delta}^{|\alpha|}}^{2}+\left\|\nabla \widetilde{\phi_{0}^{0}}\right\|_{\tilde{S}_{\delta}^{|\alpha|}}^{2}+\frac{\eta}{\delta}\right) \\
\left.+\varepsilon c\left(\left\|\nabla \widetilde{\phi_{0}^{0}}\right\|_{\delta}^{2}+\left\|\widetilde{\phi_{0}^{0}}\right\|_{\delta}^{2}+\frac{\eta}{\delta}\right) \mathrm{e}^{c c_{\star} t}\right\},
\end{gathered}
$$

are relatively dense in $\mathbf{R}(|\alpha|=0,1)$. For any $t \in[0, T]$

$$
\begin{aligned}
& \left\|\mathrm{D}_{x^{\prime}}^{\alpha} \nabla \overline{\phi^{0}}(t)\right\|_{L^{2}(\omega)}^{2} \leq\left\|\mathrm{D}_{x^{\prime}}^{\alpha} \nabla \overline{\phi_{0}^{0}}\right\|_{L^{2}(\omega)}^{2} \mathrm{e}^{-c t}, \\
& \int_{0}^{t}\left\|\mathrm{D}_{x^{\prime}}^{\alpha}, \overline{\phi^{0}}(\tau)\right\|_{L^{2}(\omega)} \mathrm{d} \tau \leq\left\|\mathrm{D}_{x^{\prime}}^{\alpha} \nabla \overline{\phi_{0}^{0}}\right\|_{L^{2}(\omega)}^{2},|\alpha|=0,1,2, \\
& \left\|\overline{n^{0}}(t)\right\|_{L^{2}(\omega)}^{2} \leq\left\|\overline{n_{0}^{0}}\right\|_{L^{2}(\omega)}^{2}+c_{\star} c t\left\|\nabla \overline{\phi_{0}^{0}}\right\|_{L^{2}(\omega)}^{2}, \\
& \left\|\mathrm{D}_{x^{\prime}}^{\alpha} \overline{n^{0}}(t)\right\|_{L^{2}(\omega)}^{2} \leq\left\|\mathrm{D}_{x^{\prime}}^{\alpha} \overline{n_{0}^{0}}\right\|_{L^{2}(\omega)}^{2}+c_{\star} c t \\
& \left\|\mathrm{D}_{x^{\prime}}^{\alpha} \overline{\phi_{0}^{0}}\right\|_{L^{2}(\omega)}^{2},|\alpha|=1,2,3, \\
& \left\|\frac{\partial \mathrm{D}_{x^{\prime}}^{\alpha} \overline{n^{0}}}{\partial t}(t)\right\|_{L^{2}(\omega)}^{2} \leq c_{\star}\left\|\mathrm{D}_{x^{\prime}}^{\alpha} \overline{\phi_{0}^{0}}\right\|_{L^{2}(\omega)}^{2} \quad|\alpha|=0,1,2 .
\end{aligned}
$$

Here $c$ is a constant independent of $\delta$, but may depends on $c_{\star}$.

By the standard arguments in help of the a priori estimates in Lemma 3.8 the solution $\phi^{0}$ established in $\S 3.1$ can be extended to any time interval $[0, T]$. Since $(1.7)_{1}-(1.7)_{2}$ are linear equations, we can easily prove Stepanov-almost-periodicity of the solution. Thus the proof of Theorem 1.2 is complete.

\section{Proof of Theorem 1.3}

Subtracting (1.5) from (1.3) and denoting by $\Phi \equiv \phi^{\varepsilon}-\phi^{0}, N \equiv n^{\varepsilon}-n^{0}$, we have 


$$
\left\{\begin{array}{l}
\frac{\partial}{\partial t}(\Delta \Phi-N)+(\nabla \Phi \times \vec{e}) \cdot \nabla \log n^{*}=c_{2} \Delta^{2} \Phi \\
\varepsilon \frac{\partial N}{\partial t}=-\varepsilon\left(\frac{\partial \tilde{n}^{0}}{\partial t}-\left(\nabla \widetilde{\phi^{0}} \times \vec{e}\right) \cdot \nabla \log n^{*}\right) \\
-\frac{1}{\bar{n}} \frac{\partial^{2}(\widetilde{\Phi}-\tilde{N})}{\partial x_{3}^{2}}+\varepsilon(\nabla \Phi \times \vec{e}) \cdot \nabla \log n^{*} \quad x \in \Omega, t>0, \\
\Phi(x, 0)=\phi_{0}^{\varepsilon}-\phi_{0}^{0}, \quad N(x, 0)=n_{0}^{\varepsilon}-n_{0}^{0} \quad \text { for } x \in \Omega, \\
\Phi(x, t)=\Delta \Phi(x, t)=N(x, t)=0 \quad \text { for } x \in \Gamma, T>t>0 .
\end{array}\right.
$$

It is easily seen that $(4.1)_{1}-(4.1)_{2}$ is equivalent to

$$
\left\{\begin{array}{l}
\frac{\partial}{\partial t}(\Delta \widetilde{\Phi}-\widetilde{N})+(\nabla \widetilde{\Phi} \times \vec{e}) \cdot \nabla \log n^{*}=c_{2} \Delta^{2} \widetilde{\Phi} \\
\varepsilon \frac{\partial \widetilde{N}}{\partial t}=-\varepsilon\left(\frac{\partial \widetilde{n^{0}}}{\partial t}-\left(\nabla \widetilde{\phi^{0}} \times \vec{e}\right) \cdot \nabla \log n^{*}\right) \\
-\frac{1}{\bar{n}} \frac{\partial^{2}(\widetilde{\Phi}-\widetilde{N})}{\partial x_{3}^{2}}+\varepsilon(\nabla \widetilde{\Phi} \times \vec{e}) \cdot \nabla \log n^{*} \\
\frac{\partial \Delta \bar{\Phi}}{\partial t}=c_{2} \Delta^{2} \bar{\Phi} \\
\frac{\partial \bar{N}}{\partial t}-(\nabla \bar{\Phi} \times \vec{e}) \cdot \nabla \log n^{*}=0 \quad \text { for } x \in \Omega, t>0 .
\end{array}\right.
$$

By virtue of Lemmas 2.3-2.5 and 3.8, the following lemma is derived from the problem for (4.2). Here we denote by $c$ a constant independent of $t$ and by $C(t)$ a constant dependent on both $t$ and the bounds of $\phi^{\varepsilon}, n^{\varepsilon}, \phi^{0}, n^{0}$, which may differ at each occurrence.

LEMMA 4.1. Let $c_{\star} \equiv \sup _{x^{\prime} \in \omega}\left|\nabla \log n^{*}\left(\left|x^{\prime}\right|\right)\right|$, and $\delta>3 c_{\dagger}^{2}\left(c_{\dagger}\right.$ is a constant of Lemma 2.2). For any $t \in[0, T], \eta>0$ the sets

$$
\begin{gathered}
\left\{\delta \in \mathbf{R} \mid \varepsilon\left(\|\nabla \widetilde{\Phi}(t)\|_{\delta}^{2}+\|\widetilde{N}(t)\|_{\delta}^{2}+\|\| \widetilde{\Phi} \|_{t, \delta}^{2}\right)+\left\|\partial_{3}(\widetilde{\Phi}-\widetilde{N})\right\|_{t, \delta}^{2}\right. \\
\left.\leq \varepsilon C(t)\left(\|\nabla \widetilde{\Phi}(0)\|_{\delta}^{2}+\|\widetilde{N}(0)\|_{\delta}^{2}+\frac{\eta}{\delta}+\varepsilon\right) \mathrm{e}^{c c \star t}\right\}
\end{gathered}
$$




$$
\begin{aligned}
& \left\{\delta \in \mathbf{R} \mid \varepsilon\left(\left\|\mathrm{D}_{x}^{\alpha} \Delta \widetilde{\Phi}(t)\right\|_{\delta}^{2}+\left\|\mathrm{D}_{x}^{\alpha} \nabla \widetilde{N}(t)\right\|_{\delta}^{2}+\| \mathrm{D}_{x}^{\alpha} \nabla \Delta \widetilde{\Phi}||_{t, \delta}^{2}\right)\right. \\
& +\left\|\mid \mathrm{D}_{x}^{\alpha} \nabla \partial_{3}(\widetilde{\Phi}-\tilde{N})\right\|_{t, \delta}^{2} \leq \varepsilon C(t)\left(\|\Delta \widetilde{\Phi}(0)\|_{\tilde{S}_{\delta}^{|\alpha|}}^{2}+\|\nabla \widetilde{N}(0)\|_{\tilde{S}_{\delta}^{|\alpha|}}^{2}+\frac{\eta}{\delta}+\varepsilon\right) \\
& \left.+\varepsilon C(t)\left(\|\nabla \widetilde{\Phi}(0)\|_{\delta}^{2}+\|\widetilde{N}(0)\|_{\delta}^{2}+\frac{\eta}{\delta}+\varepsilon\right) \mathrm{e}^{c c \star t}\right\} \\
& \left\{\delta \in \mathbf{R} \mid\left\|\partial_{\tau}(\Delta \widetilde{\Phi}-\widetilde{N})\right\| \|_{t, \delta}^{2} \leq \varepsilon C(t)\left(\|\Delta \widetilde{\Phi}(0)\|_{\tilde{S}_{\delta}^{1}}^{2}+\|\nabla \widetilde{N}(0)\|_{\tilde{S}_{\delta}^{1}}^{2}+\frac{\eta}{\delta}+\varepsilon\right)\right. \\
& \left.+\varepsilon C(t)\left(\|\nabla \widetilde{\Phi}(0)\|_{\delta}^{2}+\|\widetilde{N}(0)\|_{\delta}^{2}+\frac{\eta}{\delta}+\varepsilon\right) \mathrm{e}^{c c \star t}\right\} \\
& \left\{\delta \in \mathbf{R} \mid\left\|\partial_{\tau} \tilde{N}\right\|_{t, \delta}^{2} \leq \varepsilon C(t)\left(\|\Delta \widetilde{\Phi}(0)\|_{\delta}^{2}+\|\nabla \widetilde{N}(0)\|_{\delta}^{2}+\frac{\eta}{\delta}+\varepsilon\right)\right. \\
& \left.+\varepsilon C(t)\left(\|\nabla \widetilde{\Phi}(0)\|_{\delta}^{2}+\|\widetilde{N}(0)\|_{\delta}^{2}+\frac{\eta}{\delta}+\varepsilon\right) \mathrm{e}^{c c \star t}\right\}
\end{aligned}
$$

are relatively dense in $\mathbf{R}(|\alpha|=0,1)$. For any $t \in[0, T]$

$$
\begin{aligned}
& \left\|\mathrm{D}_{x^{\prime}}^{\alpha} \nabla \bar{\Phi}(t)\right\|_{L^{2}(\omega)}^{2} \leq\left\|\mathrm{D}_{x^{\prime}}^{\alpha} \nabla \bar{\Phi}(0)\right\|_{L^{2}(\omega)}^{2} \mathrm{e}^{-c t}, \\
& \int_{0}^{t}\left\|\mathrm{D}_{x^{\prime}}^{\alpha} \Delta \bar{\Phi}(\tau)\right\|_{L^{2}(\omega)} \mathrm{d} \tau \leq\left\|\mathrm{D}_{x^{\prime}}^{\alpha} \nabla \bar{\Phi}(0)\right\|_{L^{2}(\omega)}^{2}, \quad|\alpha|=0,1,2, \\
& \|\bar{N}(t)\|_{L^{2}(\omega)}^{2} \leq\|\bar{N}(0)\|_{L^{2}(\omega)}^{2}+c_{\star} c t\|\nabla \bar{\Phi}(0)\|_{L^{2}(\omega)}^{2}, \\
& \left\|\mathrm{D}_{x^{\prime}}^{\alpha} \bar{N}(t)\right\|_{L^{2}(\omega)}^{2} \leq\left\|\mathrm{D}_{x^{\prime}}^{\alpha} \bar{N}(0)\right\|_{L^{2}(\omega)}^{2}+c_{\star} c t\left\|\mathrm{D}_{x^{\prime}}^{\alpha} \bar{\Phi}(0)\right\|_{L^{2}(\omega)}^{2}, \quad|\alpha|=1,2,3, \\
& \left\|\frac{\partial \mathrm{D}_{x^{\prime}}^{\alpha} \bar{N}}{\partial t}(t)\right\|_{L^{2}(\omega)}^{2} \leq c_{\star}\left\|\mathrm{D}_{x^{\prime}}^{\alpha} \bar{\Phi}(0)\right\|_{L^{2}(\omega)}^{2} \quad|\alpha|=0,1,2 .
\end{aligned}
$$

Here $c$ is a constant independent of $\varepsilon$ and $\delta$, but may depends on $c_{\star}$.

From Lemma 4.1, it is easy to see that if the initial data $\left(\phi_{0}^{\varepsilon}, n_{0}^{\varepsilon}\right) \rightarrow$ $\left(\phi_{0}^{0}, n_{0}^{0}\right)$ as $\varepsilon \rightarrow 0$ in $\widetilde{S}^{3}(\omega) \times \widetilde{S}^{2}(\omega)$, then $\left(\phi^{\varepsilon}, n^{\varepsilon}\right) \rightarrow\left(\phi^{0}, n^{0}\right)$ as $\varepsilon \rightarrow 0$ in $L^{2}\left(0, T ; \widetilde{S}^{4}(\omega)\right) \times \widetilde{S}^{2,1}\left(\omega_{T}\right)$ and $\Delta \phi^{\varepsilon}-n^{\varepsilon} \rightarrow \Delta \phi^{0}-n^{0}$ as $\varepsilon \rightarrow 0$ in $\widetilde{S}^{2,1}\left(\omega_{T}\right)$. Thus the proof of Theorem 1.3 is complete.

Acknowledgments. The author would like to thank the anonymous referee for useful and kind comments. 


\section{REFERENCES}

[1] L. Amerio - G. Prouse, Almost-periodic functions and functional equations, Van Nostrand Reinhold Company, New York, 1971.

[2] J. Andres - A. M. Bersani - R. F. Grande, Hierarchy of almost-periodic function spaces, Rend. Mat. Appl., VII. Ser., 26 (2006), pp. 121-188.

[3] L. C. Berselli - M. Romito, On Leray's problem for almost periodic flows, J. Math. Sci. Univ. Tokyo, 19 (2012), pp. 69-130.

[4] A. S. Besicovitch, On generalized almost periodic functions, Proc. L. M. S., 2 (1926), pp. 495-512.

[5] A. S. Besicovitch, Almost periodic functions, Dover Publ. Inc., New York, 1955.

[6] A. S. Besicovitch - H. BoHR, Some remarks on generalisations of almost periodic functions, Meddelelser Kbenhavn, 8 (1927), pp. 1-33.

[7] S. Bochner, Abstrakte fastperiodische Funktionen, Acta Math., 61 (1933), pp. $149-184$.

[8] S. Bochner, Fastperiodische Lösungen der Wellengleichung, Acta Math., 62 (1934), pp. 227-237.

[9] H. BoHR, Zur Theorie der fastperiodischen Funktionen. I, Acta Math., 45 (1924), pp. 29-127; II, Ibid., 46 (1925), pp. 101-214; III, Ibid., 47 (1926), pp. 237-281.

[10] H. BoHr, Almost periodic functions, Chelsea, New York, 1947.

[11] G. Bruno - R. Grande - R. IAnnacci, Almost-periodic multipliers, Acta Appl. Math., 65 (2001), pp. 137-151.

[12] R. Cooke, Almost-periodic functions, Am. Math. Mon., 88 (1981), pp. 515-526.

[13] C. Corduneanu, Almost periodic functions, 2nd Engl. ed., Chelsea, New York, 1989.

[14] R. Doss, Contribution to the theory of almost-periodic functions, Ann. Math., 46 (1945), pp. 196-219.

[15] C. FoIAs, Essais dans l'étude des solutions des équations de Navier-Stokes dans l'espace. L'unicité et la presque-périodicité des solutions petites, Rend. Sem. Mat. Univ. Padova, 32 (1962), pp. 261-294.

[16] Ö. D. GÜRCAN - P. H. DIAMOND - T. S. HAHM, Radial transport of fluctuation energy in a two-field model of drift-wave turbulence, Phys. Plasmas, 13 (2006), article 052306.

[17] A. Hasegawa - K. Mima, Stationary spectrum of strong turbulence in magnetized plasma, Phys. Rev. Lett., 39 (1977), pp. 205-208.

[18] A. Hasegawa - K. Mima, Pseudo-three-dimensional turbulence in magnetized nonuniform plasma, Phys. Fluids, 21 (1978), pp. 87-92.

[19] A. Hasegawa - M. WaKatani, Plasma edge turbulence, Phys. Rev. Lett., 50 (1983), pp. 682-686.

[20] A. Hasegawa - M. Wakatani, A collisional drift wave description of plasma edge turbulence, Phys. Fluids, 27 (1984), pp. 611-618.

[21] S. Kondo - A. TANI, Initial boundary value problem for model equations of resistive drift wave turbulence, SIAM J. Math. Anal., 43 (2011), pp. 925-943.

[22] S. Kondo - A. TANI, Initial boundary value problem of Hasegawa-Wakatani equations with vanishing resistivity, Adv. Math. Sci. Appl., 21 (2011), pp. 223253. 
[23] S. Kondo - A. TANI, On the Hasegawa-Wakatani equations with vanishing resistivity, Proc. Japan Acad., 87 (2011), pp. 156-161.

[24] S. Kondo - A. TANI, Almost-periodic solutions to initial boundary value problem for model equations of resistive drift wave turbulence, Ann. Scuola Norm. Sup. Pisa, to appear.

[25] O. A. LADYZhenskaya, The boundary value problems of mathematical physics, Springer-Verlag, New York, 1985.

[26] O. A. Ladyzhenskaya - V. A. Solonnikov - N. N. URal'ceva, Linear and quasi-linear equations of parabolic type, Amer. Math. Soc., Providence, RI, 1968.

[27] G. T. LaVarnway - R. Cooke, A characterization of Fourier series of Stepanov almost-periodic functions, J. Fourier Anal. Appl., 7 (2001), pp. $127-142$.

[28] P. Marcati - A. Valli, Almost-periodic solutions to the Navier-Stokes equations for compressible fluids, Boll. Un. Mat. Ital., VI. Ser., B4 (1985), pp. 969-986.

[29] V. S. Mikhailenko - V. V. Mikhailenko - K. N. Stepanov - N. A. AzARENKOV, Renormalized non-modal theory of turbulence of plasma shear flows, Plasma Phys., 17 (2011), pp. 41-43.

[30] S. Mizohata, Partial differential equations, Iwanami, Tokyo 1965. (in Japanese)

[31] G. Prouse, Soluzioni quasi-periodiche dell'equazione differenziale di Navier-Stokes in due dimensioni, Rend. Sem. Mat. Univ. Padova, 33 (1963), pp. 186-212.

[32] G. Prouse, Almost-periodic solutions of Navier-Stokes equations and inequalities, Math.-Fys. Medd., K. Dan. Vidensk. Selsk., 42 (1989), pp. 119123.

[33] V. A. Solonnikov, On boundary value problems for linear parabolic systems of differential equations of general form, Trudy Math. Inst. Steklov, 83 (1965), pp. 3-163; English translation: Proc. Steklov Inst. Math., 83 (1965), pp. 1-184.

[34] V. V. Stepanov, Sur quelques généralisations des fonctions presque périodiques, C. R. Acad. Sci. Paris, 181 (1925), pp. 90-92.

[35] V. V. STEPANOV, Über einige Verallgemeinerungen der fastperiodischen Funktionen, Math. Ann., 95 (1926), pp. 473-498.

[36] J. Wesson, Tokamaks, Oxford Univ. Press, New York, 1987.

[37] N. WIENER, On the representation of functions by trigonometrical integrals, Math. Zeitschr., 24 (1925), pp. 575-616.

[38] S. ZaIdman, Almost-periodic functions in abstract spaces, Res. Notes in Math., 126, Pitman, Boston, 1985.

Manoscritto pervenuto in redazione il 19 Dicembre 2013. 
